\title{
Physical States in Matter-Coupled Dilaton Gravity*
}

\author{
D. Cangemi \\ Department of Physics, University of California, Los Angeles \\ 405 Hilgard Ave., Los Angeles, CA 90095-1547 \\ R. Jackiw \\ and \\ B. Zwiebach \\ Center for Theoretical Physics, Laboratory for Nuclear Science and Department of Physics \\ Massachusetts Institute of Technology, Cambridge, MA 02139-4307
}

\begin{abstract}
We revisit the quantization of matter-coupled, two-dimensional dilaton gravity. At the classical level and with a cosmological term, a series of field transformations leads to a set of free fields of indefinite signature. Without matter the system is represented by two scalar fields of opposite signature. With a particular quantization for the scalar with negative kinetic energy, the system has zero central charge and we find some physical states satisfying all the Virasoro conditions. With matter, the constraints cannot be solved because of the Virasoro anomaly. We discuss two avenues for consistent quantization: modification of the constraints, and BRST quantization. The first avenue appears to lead to very few physical states. The second, which roughly corresponds to satisfying half of the Virasoro conditions, results in a rich spectrum of physical states. This spectrum, however, differs significantly from that of free matter fields propagating on flat two-dimensional space-time.
\end{abstract}

${ }^{*}$ This work is supported in part by funds provided by the U.S. National Science Foundation (N.S.F.) under contract \#PHY-92-18990 (DC) and in part by the U.S. Department of Energy (D.O.E.) under contract \#DE-FC02-94ER40818 (RJ and BZ).

UCLA/95/TEP/16, MIT-CTP-2436 Submitted to: Annals Of Physics

May 1995 


\section{INTRODUCTION AND SUMMARY}

In the last few years much research has been carried out on models of gravity in two space-time dimensions — lineal gravity — with the hope of illuminating some puzzles of physical quantum gravity in four dimensions: the problem of time, the problem of quantizing a diffeomorphism invariant theory, the paradoxes associated with the occurrence of black holes in the classical theory and Hawking radiation in the semi-classical analysis, etc.

When modeling lineal gravity, two-dimensional analogs of Einstein's equation and of the Hilbert-Einstein action cannot be used, because they are vacuous in that dimensionality; therefore gravitational dynamics has to be invented afresh. The models that have been studied recently posit local dynamics for the "gravity" sector, which involves metric variables (metric tensor $g_{\mu \nu}, Z$ weibein $e_{\mu}^{a}$, spin-connection $\omega_{\mu}$ ) and an additional world scalar ("dilaton" or Lagrange multiplier field). Such "scalar-tensor" theories, introduced a decade ago [1], are obtained by dimensional reduction from higher-dimensional Einstein theory [1,2]. They should be contrasted with models where quantum fluctuations of matter variables induce gravitational dynamics [3], which therefore are nonlocal and do not appear to offer any insights into the questions posed by the physical, four-dimensional theory.

Another lineal gravity theory is provided by two-dimensional string theory, which is constructed in a rather indirect fashion as a field theory on a background two-dimensional spacetime. A manifestly background-independent formulation is not yet known, in contrast to the case for the simpler field theories to be discussed here. Therefore the issue of summing over space-time metrics is unclear. One would expect the semi-classical limit of two-dimensional string theory to describe, roughly speaking, nontrivial dynamics for a massless scalar field on a two-dimensional background space-time, lacking translational invariance [4].

The model we study is the so called "string-inspired dilaton gravity" - CGHS theory [5]. Initially presented in variables that arise naturally from string theory, the dilaton gravity action reads

$$
\bar{I}_{\text {gravity }}=\int d^{2} x \sqrt{-\bar{g}} e^{-2 \phi}\left(\bar{R}+4 \bar{g}^{\mu \nu} \partial_{\mu} \phi \partial_{\nu} \phi-\lambda\right)
$$

where $\phi$ is the dilaton field, $\bar{R}$ is the scalar curvature constructed from the metric tensor 
$\bar{g}_{\mu \nu}\left(\operatorname{det}\left\{\bar{g}_{\mu \nu}\right\} \equiv \bar{g}\right)$ and $\lambda$ is a cosmological constant. The over-bar distinguishes metric variables in the initial formulation from rescaled ones, which we use henceforth, that are defined by

$$
\begin{gathered}
\eta=e^{-2 \phi}, \\
g_{\mu \nu}=e^{-2 \phi} \bar{g}_{\mu \nu},
\end{gathered}
$$

in terms of which the action (1.1) becomes

$$
I_{\text {gravity }}=\int d^{2} x \sqrt{-g}(\eta R-\lambda) .
$$

We minimally couple a matter field $\varphi$,

$$
I_{\text {matter }}=\frac{1}{2} \int d^{2} x \sqrt{-g} g^{\mu \nu} \partial_{\mu} \varphi \partial_{\nu} \varphi,
$$

where, for simplicity we consider a single massless scalar field. The coupling is conformally invariant and consequently insensitive to the field redefinition (1.2b). Nevertheless, as is well known, quantum mechanically there is an anomaly. (One may increase the number of matter fields $\varphi \rightarrow \varphi^{i}, i=1, \ldots, N$, but this is of no significance to us; we do not consider the large- $N$ limit.)

The total action for the matter-coupled dilaton gravity is the sum of (1.3) and (1.4), weighted by the gravitational coupling constant $G$,

$$
I=\frac{1}{4 \pi G} I_{\text {gravity }}+I_{\text {matter }} .
$$

We have previously analyzed this model within its equivalent, Poincaré gauge invariant formulation [6]. Guided by its constraint structure, which in turn is a consequence of gauge invariance, we solved some constraints and passed to new variables by various redefinitions and canonical transformations, arriving at variables that enter quadratically and decouple from each other [6]. Our final Lagrange density reads

$$
\mathcal{L}_{g+m}=\pi_{a} \dot{r}^{a}+\Pi \dot{\varphi}-u \mathcal{E}-v \mathcal{P},
$$

where $\left\{\pi_{a}, r^{a}\right\}$ and $\{u, v\}$ is what is left of the "gravity" variables and the remaining energy $\mathcal{E}$ and momentum $\mathcal{P}$ constraints read 


$$
\begin{aligned}
& \mathcal{E}=-\frac{1}{2}\left(\frac{1}{\Lambda} \pi^{a} \pi_{a}+\Lambda r^{a \prime} r_{a}^{\prime}\right)+\frac{1}{2}\left(\Pi^{2}+\varphi^{\prime 2}\right) \\
& \mathcal{P}=-\pi_{a} r^{a \prime}-\Pi \varphi^{\prime}
\end{aligned}
$$

with $\Lambda \equiv \lambda / 8 \pi G$, and dot (dash) indicating differentiation with respect to time $t$ (space $\sigma)$. Note that the dynamically active gravitational variables $\left\{\pi_{a}, r^{a}\right\}$ enter with an indefinite quadratic form, regardless of sign $[\Lambda]: \frac{1}{\Lambda} \pi^{a} \pi_{a}+\Lambda r^{a \prime} r_{a}^{\prime}=\frac{1}{\Lambda}\left(\pi^{0}\right)^{2}-\frac{1}{\Lambda}\left(\pi^{1}\right)^{2}+\Lambda\left(r^{0^{\prime}}\right)^{2}-\Lambda\left(r^{1^{\prime}}\right)^{2}$. That there should be only two dynamical gravity variables in this theory is consistent with the familiar observation that two-dimensional dilaton gravity involves the Liouville (Weyl factor) and dilaton degrees of freedom.

An important point should be made. It is well-known [7] that a set of field redefinitions transforms the dilaton gravity CGHS model without cosmological constant to an indefinite sign quadratic form like in (1.7a). The cosmological constant is then re-inserted as an approximate conformal perturbation. In our reduction, involving field redefinitions that are local in time but not in space, a quadratic indefinite form is the entire expression, even in the presence of a cosmological constant. As an alternative to the previous gauge theoretic argument [6], we shall present a derivation of (1.6) and (1.7) within the conventional metric formalism [8].

At this stage the development encounters a quantum obstruction: the constraints that $\mathcal{E}$ and $\mathcal{P}$ should vanish appear first class on the classical level - they close under Poisson bracketing — but upon quantization they acquire a center and become second class, owing to the well known triple-derivative Schwinger term in the $[\mathcal{E}, \mathcal{P}]$ commutator, which is the same as the Virasoro anomaly. The quantum theory is recognized to be anomalous [6]. Much of our paper deals with various issues concerning this anomaly. Indeed, the central points in this paper concern answers to the following questions:

(Q1) In pure dilaton gravity theory, i.e., without matter fields, the effective dynamics is that of two scalar fields of opposite signature. In conformal field theory, such a system is usually considered to have a non-vanishing central term of $c=1+1=2$, with each scalar contributing unity, regardless of its signature. If that is the case, how is it possible that there are solutions to the constraints, as found in Refs. [6,9] ? 
(Q2) In string theory, one is not familiar with states annihilated by all Virasoro operators. Typically, states are annihilated by half of the Virasoro operators. Starting from the wave functionals of Ref. [6], one can construct the corresponding states in the explicitly regulated language provided by an oscillator expansion. Do these states satisfy all the Virasoro conditions? How many such states are there?

(Q3) When matter is coupled, the constraints have a center and cannot be solved. Is it possible to modify the constraints and eliminate the center? What are then the physical states? How does this compare with the BRST quantization of the system?

(Q4) Is there a quantization scheme where the spectrum of physical states in matter-coupled dilaton gravity is in rough agreement with the spectrum of physical states for free matter fields propagating in flat two-dimensional space-time?

As a way to summarize our results, we now sketch the answers to the above questions.

(A1) In the quantization scheme of Refs. [6,9] the scalar with negative kinetic energy is treated in a way different from what is common in conformal theory. When quantizing such a scalar, one has the choice of either positive energy states and negative norms, or negative energy states with positive norms. For a scalar with positive kinetic term one achieves both positive norms and positive energies by identifying annihilation operators with positive energy solutions and creation operators with negative energy solutions. This choice is also the usual choice in conformal theory for scalars with negative kinetic energy; it leads to positive energies, negative norms (thus the name "negative norm" scalar) and a central charge of $(+1)$. The opposite choice of creation and annihilation operators leads to negative energies, positive norms, and a central charge of $(-1)$. Therefore, there is a quantization scheme where a scalar with positive kinetic energy and a scalar with negative kinetic energy define a system with total central charge zero.

(A2) We construct explicitly the Fock space representation of the two states whose wave functionals are given in Refs. [6,9]. The two states are exponentials of bilinears of creation operators acting on the vacuum, differing by an overall sign in the exponential. We have verified explicitly that they are annihilated by all the Virasoro operators $\mathcal{L}_{n}$. Furthermore, 
we find two more states that are annihilated by all the $\mathcal{L}_{n}$ 's, but curiously, they cannot be represented by wave functionals.

(A3) We discuss two ways of modifying the constraints in order to obtain a system with zero center and then we consider the alternative provided by BRST quantization. In the first way to modify the constraints, following some ideas of K. Kuchař [10], we pass to yet further gravitational variables and then find a simple addition that can be made to $\mathcal{E} \pm \mathcal{P}$, which cancels the anomaly. This addition is related to structures that have appeared elsewhere in the literature [11], and we explain this. Physical states may exist but seem difficult to obtain. In the second way to modify the constraints, we follow standard conformal field theory lore and add a background charge to the scalar with negative kinetic energy . This can be used to reduce the central charge of this scalar to any desired value, and in particular to the value that will give zero total central charge. Despite having zero central charge, it seems unlikely that there are many physical states. In the second way, using the BRST procedure, the ghost system is coupled and it carries a center (-26). Furthermore, for the positive-sign scalar of the gravitational sector a background charge is used to increase its center so that together with the negative sign scalar [which here gives center $(+1)$ ] and matter, one attains the center $(+26)$. Physical states are those states that are annihilated by the BRST operator and cannot be written as the BRST operator acting on another state. Roughly speaking, BRST quantization is a consistent procedure to impose half of the constraints. The spectrum of physical states for our present problem is rich and follows from well-known results [12, 13].

(A4) The procedure of modifying the constraints to get zero center, and then imposing all of them seems unable to produce a large (or infinite) number of physical states. In the BRST method the space-time is taken to be a flat cylinder, and we get a set of physical states that is not very different from that one would expect in the zero gravity limit. The continuous parameters that describe the states, however, do not work out. For a single scalar field we should have one continuous parameter, the vacuum "momentum", but here we obtain two continuous parameters. We feel that a large mismatch between these sets of physical states is an indication that the semi-classical approximation to quantized gravity may be 
problematic.

Let us describe briefly the organization of this paper. In Section \ we review the gauge theoretical formulation of the model. In Section III we explain how to reduce the theory in terms of metric-based variables, to indefinite sign quadratic form [8]. Section IV] discusses the modification of the constraints to achieve zero central term as in Ref. [10]. In Section $\square$, we describe the alternative quantizations of the scalar field with negative kinetic energy. We consider the pure dilaton gravity theory and give the oscillator description of the physical states, showing that they satisfy all the Virasoro conditions. In Section VI we show how a standard conformal improvement of the negative norm scalar gives a constraint system with zero center. In Section VII we describe the BRST quantization of dilaton gravity with and without matter, and compare the spectrum of physical states to that of the flat-space limit. Finally, in Section VIII we offer some general comments, discuss open questions, and speculate about the four-dimensional theory, using the insights drawn from the 2-dimensional toy model.

\section{GAUGE THEORETICAL STARTING POINT}

This Section has two parts. In the first part we summarize the gauge theoretical formulation of the dilaton gravity theory and describe the constraint structure of the theory. In the second part, for the benefit of the interested reader, we give a self-contained derivation of most of the results quoted in the first part.

\section{A. Summary of the Gauge Formulation}

The pure dilaton gravity theory given in (1.3), and the matter-coupled dilaton gravity theory in (1.4), (1.5) can be given a gauge theoretical formulation. The basic idea is to work with Einstein-Cartan variables - Zweibein and spin-connection - and to view them as gauge potentials for some suitably chosen Lie group. Specifically, one combines the EinsteinCartan variables into a Lie algebra valued gauge connection $A_{\mu}$, builds the gauge curvature 
$F_{\mu \nu}=\partial_{\mu} A_{\nu}-\partial_{\nu} A_{\mu}+\left[A_{\mu}, A_{\nu}\right]$, introduces a non-singular, bilinear invariant on the Lie algebra $\langle\mid\rangle$, and forms the scalar-tensor action

$$
I \propto \int\left\langle H \mid F_{\mu \nu} d x^{\mu} d x^{\nu}\right\rangle
$$

where $H$ comprises (a multiplet of) Lagrange multipliers. (The invariant bilinear $\langle\mid\rangle$ is constructed from the Cartan-Killing metric when the group is semi-simple; otherwise alternative expressions must be used.) The dynamical equations involve $H$ and $A_{\mu}$; when they are re-expressed in terms of the dilaton field (which corresponds to one component of the $H$ multiplet) and in terms of the metric tensor (which is reconstructed from the Zweibein and spin-connection that are collected in $A_{\mu}$ ) one regains the metric version of the gravitational equations.

The action (2.1) does not depend on a background metric, and the hoped-for advantage in the gauge theoretical formulation of a geometric gravity theory is that one can use quantization methods that have been perfected during the decades spent studying gauge theories, thereby circumventing some of the obstacles to quantizing a gravity theory. Moreover, enforcing gauge invariance can resolve ambiguities and uncertainties in the quantization procedure. Of course, when the gauge theoretical approach succeeds, it should also instruct, in retrospect, how the goal could have been reached if one remained with the conventional formalism.

The theory given in (1.3) is formulated using the centrally extended, 4-parameter Poincaré group [14]. In the algebra of this group, there occur two translation generators $P_{a},(a=0,1)$ whose commutator contains the central element $I$,

$$
\left[P_{a}, P_{b}\right]=\epsilon_{a b} I
$$

while the commutator with the Lorentz rotation generator $J$ is unmodified.

$$
\left[P_{a}, J\right]=\epsilon_{a}^{b} P_{b}
$$

[Notation: tangent space indices $(a, b, \ldots)$ are moved with $h_{a b} \equiv \operatorname{diag}(1,-1) ; \epsilon^{a b}=-\epsilon^{b a}$, $\epsilon^{01}=1$; space-time points $x^{\mu}$ are 2 -vectors $\left.(t, \sigma).\right]$ The Zweibein $e_{\mu}^{a}$ is taken to be the gauge 
potential for translations, the spin-connection $\omega_{\mu}$ is the gauge potential for the Lorentz rotation, and a further $\mathrm{U}(1)$ gauge potential $a_{\mu}$ has to be introduced for the central element I. Thus, the gauge connection is constructed as

$$
A_{\mu}=e_{\mu}^{a} P_{a}+\omega_{\mu} J+a_{\mu} I
$$

and the curvature reads

$$
\begin{aligned}
F & =\frac{1}{2} \epsilon^{\mu \nu} F_{\mu \nu}=\epsilon^{\mu \nu}\left\{\left(\partial_{\mu} e_{\nu}^{a}+\epsilon_{b}^{a} \omega_{\mu} e_{\nu}^{b}\right) P_{a}+\partial_{\mu} \omega_{\nu} J+\left(\partial_{\mu} a_{\nu}+\frac{1}{2} e_{\mu}^{a} \epsilon_{a b} e_{\nu}^{b}\right) I\right\} \\
& \equiv f^{a} P_{a}+f^{2} J+f^{3} I .
\end{aligned}
$$

Correspondingly, a quartet multiplet of Lagrange multiplier fields is introduced

$$
H=\eta_{a} h^{a b} P_{b}-\eta_{3} J-\eta_{2} I
$$

and the invariant, non-degenerate bilinear is given by

$$
\langle H \mid F\rangle=\eta_{a} f^{a}+\eta_{2} f^{2}+\eta_{3} f^{3}
$$

thus providing a Lagrange density $\mathcal{L}$ for the action $(2.1)$. It follows that the dynamics implied by $(2.1)-(2.5)$ is the same as that of $(1.3)$ [or of $(1.1)]: \eta$ coincides with $\frac{1}{2} \eta_{2}, \sqrt{-g} R=$ $2 \epsilon^{\mu \nu} \partial_{\mu} \omega_{\nu}$ and the cosmological constant $\lambda$, absent from the gauge action, emerges as the value in the solution for $\eta_{3}$, which is also a group invariant [14].

The gauge theoretic formulation of the matter-coupled dilaton gravity theory (11.5) requires further developments [6]. Gauge invariance in the matter sector is achieved by a Higgs-like mechanism, which introduces a new field $q^{a}$ — the Poincaré coordinate — and its canonical conjugate $p_{a}$ 15, 16]. The complete gauge invariant Lagrange density for the matter-gravity system that we studied reads, in first order form [6],

$$
\mathcal{L}_{g+m}=\frac{1}{4 \pi G}\left(\eta_{a} \dot{e}_{1}^{a}+\eta_{2} \dot{\omega}_{1}+\eta_{3} \dot{a}_{1}\right)+p_{a} \dot{q}^{a}+\Pi \dot{\varphi}+e_{0}^{a} G_{a}+\omega_{0} G_{2}+a_{0} G_{3}-u \mathcal{E}-v \mathcal{P}
$$

where,

$$
\begin{aligned}
G_{a} & \equiv \frac{1}{4 \pi G}\left(\eta_{a}^{\prime}+\epsilon_{a}^{b} \eta_{b} \omega_{1}+\eta_{3} \epsilon_{a b} e_{1}^{b}\right)+\epsilon_{a}^{b} p_{b}, \\
G_{2} & \equiv \frac{1}{4 \pi G}\left(\eta_{2}^{\prime}+\eta_{a} \epsilon_{b}^{a} e_{1}^{b}\right)-q^{a} \epsilon_{a}^{b} p_{b}, \\
G_{3} & \equiv \frac{1}{4 \pi G} \eta_{3}^{\prime},
\end{aligned}
$$


and,

$$
\begin{aligned}
& \mathcal{E} \equiv(D q)^{a} \epsilon_{a}{ }^{b} p_{b}+\frac{1}{2}\left(\Pi^{2}+\varphi^{\prime 2}\right) \\
& \mathcal{P} \equiv-p_{a}(D q)^{a}-\Pi \varphi^{\prime} \\
& (D q)^{a} \equiv q^{a \prime}+\epsilon^{a}{ }_{b}\left(q^{b} \omega_{1}-e_{1}^{b}\right) .
\end{aligned}
$$

(To conform to usual conventions, some signs are changed from Ref. [6].)

The Poincaré-coordinate $q^{a}$ may be set to zero by a gauge transformation, whereupon (2.6) $-(2.9)$ can be shown to be equivalent to (1.5), as is demonstrated in the next subSection.

With dynamical $q^{a}$, (2.6)-(2.9) define a Poincaré gauge invariant system - that is why $q^{a}$ is like a Higgs field. In our previous investigation [6], we began with (2.6)-(2.9), which although not manifestly gauge invariant, clearly exhibit that symmetry by virtue of the symplectic structure in (2.6) and the vanishing of the gauge constraints $\left(G_{a}, G_{2}, G_{3}\right)$. In the next subSection, we show explicitly how the haphazard-looking expressions (2.6)-(2.9) in fact follow from a manifestly gauge invariant formalism. The constraint structure in the above equations is a guide to field redefinitions and canonical transformations [6] that map the model onto decoupled field variables entering quadratically in (1.6) and (1.7).

\section{B. Deriving the Constraint Structure}

In this subSection we present a gauge formulation of matter-coupled dilaton gravity (1.3), (1.4) and give a derivation of Eqs. (2.6)-(2.9). As mentioned before, the gauge structure is based on a central extension of the two-dimensional Poincaré algebra (2.2) with four generators $\left\{Q_{A}\right\}_{A=0,1,2,3}=\left\{P_{a}, J, I\right\}_{a=0,1}$. In the adjoint representation, a group element parametrized by $\left\{\theta^{a}, \alpha, \beta\right\}$ is a $4 \times 4$ matrix

$$
U_{B}^{A}=\left(\begin{array}{ccc}
\Lambda(\alpha)^{a}{ }_{b} & -\epsilon^{a}{ }_{c} \theta^{c} & 0 \\
0 & 1 & 0 \\
\theta^{c} \epsilon_{c d} \Lambda(\alpha)^{d}{ }_{b} & -\frac{1}{2} \theta^{c} \theta_{c} & 1
\end{array}\right),
$$


with Lorentz transformation $\Lambda(\alpha)^{a}{ }_{b}=\delta^{a}{ }_{b} \cosh \alpha+\epsilon_{b}^{a} \sinh \alpha$, and no dependence on the parameter $\beta$. A contravariant element $H=\eta^{A} Q_{A}=\eta^{a} P_{a}+\eta^{2} J+\eta^{3} I$ transforms as $\eta^{A} \rightarrow$ $\left(U^{-1}\right)_{B}^{A} \eta^{B}$, or in components

$$
\begin{aligned}
& \eta^{a} \rightarrow\left(\Lambda^{-1}\right)^{a}{ }_{b}\left(\eta^{b}+\eta^{2} \epsilon_{c}^{b} \theta^{c}\right), \\
& \eta^{2} \rightarrow \eta^{2}, \\
& \eta^{3} \rightarrow \eta^{3}+\eta^{a} \epsilon_{a b} \theta^{b}-\frac{1}{2} \eta^{2} \theta^{a} \theta_{a} .
\end{aligned}
$$

Notice that vectors along $I$ are invariant, a property of a solvable algebra.

Since the extended Poincaré algebra is not semi-simple, it has a degenerate Cartan-Killing form. There is, however, a one-parameter family of inner products

$$
\begin{gathered}
\left\langle Q_{A} \mid Q_{B}\right\rangle=h_{A B}, \\
h_{A B}=\left(\begin{array}{ccc}
h_{a b} & 0 & 0 \\
0 & c & -1 \\
0 & -1 & 0
\end{array}\right),
\end{gathered}
$$

that are invariant $\left\langle Q_{C} U^{C}{ }_{A} \mid Q_{D} U^{D}{ }_{B}\right\rangle=\left\langle Q_{A} \mid Q_{B}\right\rangle$, and non-degenerate. The inverse to $h_{A B}$ is

$$
h^{A B}=\left(\begin{array}{ccc}
h^{a b} & 0 & 0 \\
0 & 0 & -1 \\
0 & -1 & -c
\end{array}\right) \text {. }
$$

It is convenient to set the parameter $c$ to zero. This is achieved by shifting $J$ with a multiple of the central element $I$, and we make this choice henceforth. The bilinear form defines a metric on the 4-dimensional (Lie algebra) space, which is used to raise and lower indices, interchanging covariant and contravariant tensors, e.g. $\eta_{A}=h_{A B} \eta^{B}$ transforms as $\eta_{A} \rightarrow \eta_{B} U^{B}{ }_{A}$, in components

$$
\begin{aligned}
\eta_{a} & \rightarrow\left(\eta_{b}-\eta_{3} \epsilon_{b c} \theta^{c}\right) \Lambda_{a}^{b}, \\
\eta_{2} & \rightarrow \eta_{2}-\eta_{a} \epsilon_{b}^{a} \theta^{b}-\frac{1}{2} \eta_{3} \theta^{a} \theta_{a}, \\
\eta_{3} & \rightarrow \eta_{3} .
\end{aligned}
$$


The gravitational fields are collected in the gauge potential (2.3a) and the existence of the non-degenerate inner product (2.12) allows the construction of an action for pure dilaton gravity

$$
I_{\text {gravity }}=\int d^{2} x\langle H \mid F\rangle .
$$

The solutions to the classical equations of motion,

$$
A_{\mu}=U^{-1} \partial_{\mu} U, \quad \partial_{\mu}\left(U H U^{-1}\right)=0,
$$

are labeled by the two gauge invariants $M=\langle H \mid H\rangle=\eta^{a} \eta_{a}-2 \eta_{2} \eta_{3}$ and $\lambda=\langle I \mid H\rangle=\eta_{3}$. The norm square of $H$ corresponds to the "mass" $M$ of the CGHS black-hole. Its projection on the invariant central element coincides with the cosmological constant $\lambda$, which is not fixed but enters as a dynamical variable in our model.

The coupling of matter to dilaton gravity in an explicit gauge invariant way requires the use of two additional fields $q^{a}$ called Poincaré coordinates [15, 16]. Consider a fiducial quantity $q_{(0)}^{A}$ that is constant and picks out the Lorentz generator [17]

$$
q_{(0)}=q_{(0)}^{A} Q_{A}=J
$$

Using the Poincaré coordinates, we transform $q_{(0)}$ into the $P_{a}$ direction and obtain a Lie algebra element that is taken to transform contravariantly

$$
q \equiv \exp \left(q^{a} \epsilon_{a}^{b} P_{b}\right) q_{(0)} \exp \left(-q^{a} \epsilon_{a}^{b} P_{b}\right)=q^{a} P_{a}+J+\frac{1}{2} q^{a} q_{a} I .
$$

The transformation of this vector, $q^{A} \rightarrow\left(U^{-1}\right)^{A}{ }_{B} q^{B}$, induces a transformation of the Poincaré coordinates, see (2.11a),

$$
q^{a} \rightarrow\left(\Lambda^{-1}\right)^{a}{ }_{b}\left(q^{b}+\epsilon^{b}{ }_{c} \theta^{c}\right)
$$

which defines a non-linear action of the group [15]. Notice that the Poincaré coordinates can always be set to zero with an appropriate gauge transformation $\theta^{b}=-\epsilon_{c}^{b} q^{c}$.

Matter fields are described in a similar way. Although we are ultimately interested in massless fields, we find a manifestly gauge invariant action only for massive scalar fields with mass $m$, which we later set to zero. Starting with the combination 


$$
\Phi_{(0)}=\frac{\varphi^{a}}{m^{2}} P_{a}-\varphi_{3} J
$$

we construct

$$
\Phi \equiv \exp \left(q^{a} \epsilon_{a}^{b} P_{b}\right) \Phi_{(0)} \exp \left(-q^{a} \epsilon_{a}^{b} P_{b}\right)=\left(\frac{\varphi^{a}}{m^{2}}-\varphi_{3} q^{a}\right) P_{a}-\varphi_{3} J+\left(\frac{\varphi^{a}}{m^{2}}-\frac{1}{2} \varphi_{3} q^{a}\right) q_{a} I,
$$

and choose it to transform contravariantly under a gauge transformation. According to (2.11), this induces the matter field transformation law

$$
\begin{aligned}
& \varphi^{a} \rightarrow\left(\Lambda^{-1}\right)^{a}{ }_{b} \varphi^{b}, \\
& \varphi_{3} \rightarrow \varphi_{3} .
\end{aligned}
$$

Gravity is minimally coupled through the covariant derivative, and the matter-gravity interaction is taken in explicitly gauge invariant form

$$
I_{\text {matter }}=\frac{m^{2}}{4} \int d^{2} x\left\langle q \mid \epsilon^{\mu \nu}\left[D_{\mu} \Phi, D_{\nu} \Phi\right]+\frac{m^{2}}{2} e(q)[\Phi,[\Phi, q]]\right\rangle,
$$

where

$$
\begin{aligned}
D_{\mu} & =\partial_{\mu}+\left[A_{\mu}, \quad\right], \\
e(q) & =\frac{1}{2} \epsilon^{\mu \nu} \epsilon_{a b}\left(D_{\mu} q\right)^{a}\left(D_{\nu} q\right)^{b} .
\end{aligned}
$$

Together with the dilaton gravity action (2.14), (2.22) describes two-dimensional mattercoupled dilaton gravity in a manifestly topological and gauge invariant way.

The relation to the Klein-Gordon action becomes apparent if we rewrite (2.22) using the components of the fields. Up to surface terms, which we drop, $I_{\text {matter }}$ is

$$
\begin{aligned}
I_{\text {matter }}=- & \frac{1}{2} \int d^{2} x\left[\varphi_{3} \varphi_{a} f^{a}+\varphi_{a}\left(\frac{1}{2} \frac{\varphi^{a}}{m^{2}}-\varphi_{3} q^{a}\right) f^{2}\right] \\
& +\frac{1}{8} \int d^{2} x e(q)\left(\varphi^{a}+2 \epsilon^{a b} E(q)_{b}^{\mu} \partial_{\mu} \varphi_{3}\right)\left(\varphi_{a}+2 \epsilon_{a}{ }^{c} E(q)_{c}^{\nu} \partial_{\nu} \varphi_{3}\right) \\
& +\frac{1}{2} \int d^{2} x e(q)\left[h^{a b} E(q)_{a}^{\mu} E(q)_{b}^{\nu} \partial_{\mu} \varphi_{3} \partial_{\nu} \varphi_{3}-m^{2} \varphi_{3}{ }^{2}\right],
\end{aligned}
$$

with

$$
E(q)_{a}^{\mu}=\frac{1}{e(q)} \epsilon^{\mu \nu} h_{a b}\left(D_{\nu} q\right)^{b}
$$


The first term in the right side of (2.24) is absorbed in a redefinition of $\eta_{a}$ and $\eta_{2}$ in the pure dilaton gravity Lagrangian (2.5), (2.14), whereupon the massless limit can be taken. The second term sets $\varphi^{a}$ equal to the gradient of the field $\varphi_{3}$. In the gauge $q^{a}=0, e(q)$ becomes the density $\sqrt{-g}, E(q)_{a}^{\mu}$ the inverse Zweibein $E_{a}^{\mu}$ from which the metric is reconstructed: $h^{a b} E_{a}^{\mu} E_{b}^{\nu}=g^{\mu \nu}$, and the third term coincides with the action of a massive scalar field [18].

Quantization is carried out in a first order formalism and we want to show now that (2.6)-(2.9) follow from (2.14) and (2.24). As said before, the first term on the right side of (2.24) is combined with the dilaton gravity action by a redefinition of $\eta_{a}$ and $\eta_{2}$. The spatial components of the gauge fields multiply $\dot{\eta}_{A}$ and become the momenta conjugated to $\eta_{A}$, whereas the gauge field time components play the role of Lagrange multipliers. In the remainder of the first order expression for (2.24), the coefficients of $\dot{q}^{a}$ and $\dot{\varphi}_{3}$ are identified with their respective canonical momenta $p_{a}$ and $\Pi$, whose definition is enforced with three Lagrange multipliers $\left\{N^{a}, N\right\}$. Consequently, $\mathcal{L}_{g+m}=\frac{1}{4 \pi G} \mathcal{L}_{\text {gravity }}+\mathcal{L}_{\text {matter }}$ is written in an equivalent form as

$$
\begin{aligned}
\mathcal{L}_{g+m}=\frac{1}{4 \pi G}\left(\eta_{a} \dot{e}_{1}^{a}+\eta_{2} \dot{\omega}_{1}+\eta_{3} \dot{a}_{1}\right)+p_{a} \dot{q}^{a}+\pi \dot{\varphi}_{3}+e_{0}^{a} G_{a}+\omega_{0} G_{2}+a_{0} G_{3} \\
+N^{a}\left[p_{a}-\frac{1}{2} \epsilon_{a b} \varphi^{b} \varphi_{3}^{\prime}-\frac{1}{8} \epsilon_{a b}(D q)^{b}\left(\varphi^{c} \varphi_{c}-4 m^{2} \varphi_{3}^{2}\right)\right] \\
+N\left[\Pi-\frac{1}{2} \varphi^{a} \epsilon_{a b}(D q)^{b}\right],
\end{aligned}
$$

where the $\left\{G_{A}\right\}_{A=0,1,2,3}$ are given by (2.7) and $D$ denotes the spatial component of the covariant derivative (2.23). The three constraints enforced by $\left\{N^{a}, N\right\}$ may be replaced by three alternative ones, enforced by $\{u, v, w\}$,

$$
\begin{aligned}
& -u\left[-p_{a} \epsilon_{b}^{a}(D q)^{b}+\frac{1}{2}\left\{\Pi^{2}+\varphi_{3}^{\prime 2}-\left[\varphi_{3}^{\prime}+\frac{1}{2} \varphi_{a}(D q)^{a}\right]^{2}+m^{2}(D q)^{a}(D q)_{a} \varphi_{3}^{2}\right\}\right] \\
& -v\left[-p_{a}(D q)^{a}-\Pi \varphi_{3}^{\prime}\right] \\
& -w\left[p_{a}(D q)^{a}+\frac{1}{2} \varphi^{a} \epsilon_{a b}(D q)^{b} \varphi_{3}^{\prime}\right] .
\end{aligned}
$$

Since no derivative acts on the $\varphi^{a}$, we eliminate them through their equations of motion

$$
u(D q)^{a}\left(\varphi_{3}^{\prime}+\frac{1}{2} \varphi_{b}(D q)^{b}\right)-w \epsilon_{b}^{a}(D q)^{b} \varphi_{3}^{\prime}=0
$$

Successive projections of Eq. (2.27a) onto $(D q)_{a}$ and $\epsilon_{a b}(D q)^{b}$ imply 


$$
w=0, \quad \varphi_{3}^{\prime}+\frac{1}{2} \varphi_{a}(D q)^{a}=0 .
$$

In the massless case, (2.26) and (2.27b) reproduce (2.6)-(2.9), once $\varphi_{3}$ is identified with $\varphi$.

\section{METRIC-BASED STARTING POINT}

Beginning with the metric formulation of the action (1.5) as in (1.3) and (1.4), we derive (1.6) and (1.7), without using gauge theoretical ideas. To begin, make the following parameterization for the metric tensor [19].

$$
g_{\mu \nu}=e^{2 \rho}\left(\begin{array}{cc}
u^{2}-v^{2} & v \\
v & -1
\end{array}\right) .
$$

Passing to canonical form, the action (11.5) becomes

$$
I=\int d^{2} x\left[\Pi_{\rho} \dot{\rho}+\Pi_{\eta} \dot{\eta}+\Pi \dot{\varphi}-u \mathcal{E}-v \mathcal{P}\right],
$$

where the constraints are

$$
\begin{aligned}
& \mathcal{E}=-\frac{1}{2 \pi G}\left(\eta^{\prime \prime}-\rho^{\prime} \eta^{\prime}\right)+2 \pi G \Pi_{\rho} \Pi_{\eta}+\frac{\lambda}{4 \pi G} e^{2 \rho}+\frac{1}{2}\left(\Pi^{2}+\varphi^{\prime 2}\right), \\
& \mathcal{P}=-\Pi_{\rho} \rho^{\prime}+\Pi_{\rho}^{\prime}-\Pi_{\eta} \eta^{\prime}-\Pi \varphi^{\prime}
\end{aligned}
$$

The variables $\rho, \Pi_{\rho}, \eta$ and $\Pi_{\eta}$ are changed to new variables $\rho^{a}$ and $p_{a}(a=0,1)$ using the canonical transformation induced by the generating functional

$$
\begin{aligned}
F\left(\rho, \Pi_{\eta} ; \rho^{0}, \rho^{1}\right) & =-\int_{-\infty}^{\infty} d \sigma e^{\rho(\sigma)}\left[\rho^{0}(\sigma) \sinh \theta(\sigma)-\rho^{1}(\sigma) \cosh \theta(\sigma)\right] \\
\theta(\sigma) & \equiv 2 \pi G \int_{-\infty}^{\sigma} d \tilde{\sigma} \Pi_{\eta}(\tilde{\sigma}) .
\end{aligned}
$$

(All of the fields in this expression have the same time argument, which is suppressed; $\sigma$ and $\tilde{\sigma}$ are spatial variables). The new variables $\rho^{a}(\sigma)$ and $p_{a}(\sigma)$ obey $p_{a}=-\delta F / \delta \rho^{a}$, while similarly for the old variables, $\eta=-\delta F / \delta \Pi_{\eta}$ and $\Pi_{\rho}=\delta F / \delta \rho$. Note that the above canonical transformations are non-local in the spatial variable $\sigma$ but local in time.

In these variables the constraints (3.3) become

$$
\begin{aligned}
\mathcal{E} & =-\rho^{a \prime} \epsilon_{a}^{b} p_{b}-\frac{\lambda}{4 \pi G} p_{a} p^{a}+\frac{1}{2}\left(\Pi^{2}+\varphi^{\prime 2}\right), \\
\mathcal{P} & =-p_{a} \rho^{a \prime}-\Pi \varphi^{\prime}
\end{aligned}
$$


This form of the constraints appears at an intermediate step in [6]. In order to make the comparison with (1.7) complete, we make the transformation

$$
\begin{aligned}
\pi_{a} & =\frac{\lambda}{4 \pi G} p_{a}-\frac{1}{2} \epsilon_{a b} \rho^{b \prime}, \\
r^{a} & =\frac{4 \pi G}{\lambda} \rho^{a},
\end{aligned}
$$

and find that (3.6) coincide with (1.7) [8].

\section{REMOVING ANOMALIES IN THE CANONICAL THEORY}

The theory described by (1.6) and (1.7) appears to be very simple: there are three independent fields $\left\{r^{a}, \varphi\right\}$ and together with the canonical momenta $\left\{\pi_{a}, \Pi\right\}$ they lead to a quadratic Hamiltonian, which has no interaction terms among the three; see (1.7a). Similarly, the momentum comprises non-interacting terms, see (1.7b). However, there remains a subtle "correlation interaction" as a consequence of the requirement that the energy and momentum densities, $\mathcal{E}$ and $\mathcal{P}$, annihilate physical states, as follows from varying the Lagrange multipliers $u$ and $v$ in $(1.6)$

$$
\begin{gathered}
\mathcal{E}|\psi\rangle=0, \\
\mathcal{P}|\psi\rangle=0 .
\end{gathered}
$$

Thus, even though $\mathcal{E}$ and $\mathcal{P}$ each are sums of non-interacting variables, the physical states $|\psi\rangle$ are not direct products of states for the separate degrees of freedom. Note that Eqs. (4.1) comprise the entire physical content of the theory. There is no need for any further "gauge fixing" or "ghost" variables — this is the advantage of the Hamiltonian formalism that we are pursuing in this Section.

The momentum constraint (4.1b) is easy to unravel: in a Schrödinger representation where $|\psi\rangle$ is realized as a functional $\Psi$ of $\left\{r^{a}, \varphi\right\}$, on which these quantities act by multipli-

cation while the canonically conjugate momenta $\left\{\pi_{a}, \Pi\right\}$ act by (functional) differentiation, Eq. (4.1b) implies that $\Psi$ is a functional that is invariant against arbitrary reparameterization of the spatial $\sigma$-variable: $\sigma \rightarrow \tilde{\sigma}(\sigma)$. Such functionals are readily constructed. Moreover, 
the commutator of $\mathcal{P}$ with itself closes on itself - even in the quantum theory the constraint is first class and there is no obstruction to $(4.1 \mathrm{~b})$

$$
i[\mathcal{P}(\sigma), \mathcal{P}(\tilde{\sigma})]=(\mathcal{P}(\sigma)+\mathcal{P}(\tilde{\sigma})) \delta^{\prime}(\sigma-\tilde{\sigma})
$$

(The commutator is at equal times, and the time argument is suppressed.)

The constraint (4.1a) is the Wheeler-DeWitt equation, and here an anomaly obstructs solving it: while the commutator of $\mathcal{E}$ with itself closes on $\mathcal{P}$ 20

$$
i[\mathcal{E}(\sigma), \mathcal{E}(\tilde{\sigma})]=(\mathcal{P}(\sigma)+\mathcal{P}(\tilde{\sigma})) \delta^{\prime}(\sigma-\tilde{\sigma})
$$

the $[\mathcal{E}, \mathcal{P}]$ commutator possesses, in addition to an innocuous term involving $\mathcal{E}$, a quantum $c$-number anomaly

$$
i[\mathcal{E}(\sigma), \mathcal{P}(\tilde{\sigma})]=(\mathcal{E}(\sigma)+\mathcal{E}(\tilde{\sigma})) \delta^{\prime}(\sigma-\tilde{\sigma})-\frac{1}{12 \pi} \delta^{\prime \prime \prime}(\sigma-\tilde{\sigma})
$$

The triple-derivative Schwinger term converts the classical first class constraint into a quantal second class one and prevents finding a solution to (4.1a). Note that the Schwinger term arises solely from the matter terms; the Schwinger term in the gravitational variables vanishes owing to the indefinite sign: the contribution from $a=0$ cancels against that from $a=1$. Correspondingly, in the absence of matter variables, all constraints can be, and have been solved - there is no obstruction in the gravity sector.

In the absence of matter one finds two gravity states $| \pm\rangle_{\text {gravity }}$. They are explicitly represented by the functionals

$$
\Psi_{\text {gravity }}\left(r^{a}\right)=\exp \left( \pm i \frac{\Lambda}{2} \int d \sigma \epsilon_{a b} r^{a} r^{b^{\prime}}\right)
$$

and satisfy the gravitational portions of the constraints (1.7) [6]

$$
\begin{aligned}
-\frac{1}{2}\left(\frac{1}{\Lambda} \pi^{a} \pi_{a}+\Lambda r^{a \prime} r_{a}^{\prime}\right) \Psi_{\text {gravity }} & =\frac{1}{2}\left(\frac{1}{\Lambda} \frac{\delta^{2}}{\delta r^{a} \delta r_{a}}-\Lambda r^{a \prime} r_{a}^{\prime}\right) \Psi_{\text {gravity }}=0 \\
\left(-r^{a \prime} \pi_{a}\right) \Psi_{\text {gravity }} & =\left(i r^{a \prime} \frac{\delta}{\delta r^{a}}\right) \Psi_{\text {gravity }}=0 .
\end{aligned}
$$

The states (4.5) annihilated by the constraints may also be related to the Fock vacuum $|0\rangle$, which in the Schrödinger representation reads 


$$
\begin{aligned}
\Psi_{\text {vacuum }}\left(r^{a}\right) & =\operatorname{det} \frac{1}{2}\left(\frac{\Lambda \omega}{\pi}\right) \cdot \exp \left(-\frac{\Lambda}{2} \int d \sigma d \tilde{\sigma}\left(r^{0} \omega r^{0}+r^{1} \omega r^{1}\right)\right), \\
\omega(\sigma, \tilde{\sigma}) & =\int \frac{d k}{2 \pi} e^{-i k(\sigma-\tilde{\sigma})}|k|
\end{aligned}
$$

The gravity states are then given by

$$
| \pm\rangle_{\text {gravity }}=\operatorname{det}^{-\frac{1}{2}}\left(\frac{\Lambda \omega}{2 \pi}\right) \cdot\left[\exp \left( \pm \frac{\Lambda}{2} \int d k a_{0}^{\dagger}(k) \epsilon(k) a_{1}^{\dagger}(-k)\right)\right]|0\rangle
$$

where the creation operators are defined in the same way for both fields

$$
a_{a}^{\dagger}(k)=\frac{-i}{\sqrt{4 \pi|\Lambda k|}} \int d \sigma e^{i k \sigma} \pi_{a}(\sigma)+\sqrt{\frac{|\Lambda k|}{4 \pi}} \int d \sigma e^{i k \sigma} r^{a}(\sigma) .
$$

These pure dilaton gravity states, satisfying all constraints, have been obtained within the gauge theoretical formalism in Refs. [6,9], within the metric formalism in Ref. [19], and the relation between the two approaches has been elucidated in Ref. [21].

Once matter degrees of freedom are included, however, our Hamiltonian analysis of the theory cannot be taken farther owing to the Schwinger term. We now show that it is possible to alter the theory, by a change in the gravitational sector, whereupon the anomaly cancels.

To describe this modification, it is first convenient to form the sum and difference of the constraints, putting them into the decoupled, Virasoro form,

$$
\begin{aligned}
\Theta_{ \pm} & =\frac{1}{2}(\mathcal{E} \mp \mathcal{P}), \\
\mathcal{L} & =\pi_{a} \dot{r}^{a}+\Pi \dot{\varphi}-\lambda^{+} \Theta_{+}-\lambda^{-} \Theta_{-}, \\
\lambda^{ \pm} & =u \mp v,
\end{aligned}
$$

with $(4.2)-(4.4)$ becoming

$$
\begin{aligned}
& {\left[\Theta_{ \pm}(\sigma), \Theta_{ \pm}(\tilde{\sigma})\right]= \pm i\left[\Theta_{ \pm}(\sigma)+\Theta_{ \pm}(\tilde{\sigma})\right] \delta^{\prime}(\sigma-\tilde{\sigma}) \mp \frac{i}{24 \pi} \delta^{\prime \prime \prime}(\sigma-\tilde{\sigma})} \\
& {\left[\Theta_{ \pm}(\sigma), \Theta_{\mp}(\tilde{\sigma})\right]=0}
\end{aligned}
$$

Next, following Kuchař [10], we pass to new canonical variables, with the transformation

$$
\begin{gathered}
P_{ \pm}=-\frac{1}{2 \sqrt{\Lambda}}\left(\pi_{0}+\pi_{1}\right) \pm \frac{\sqrt{\Lambda}}{2}\left(r^{0 \prime}-r^{1 \prime}\right) \\
X^{ \pm \prime}= \pm \frac{1}{2 \sqrt{\Lambda}}\left(\pi_{0}-\pi_{1}\right)-\frac{\sqrt{\Lambda}}{2}\left(r^{0 \prime}+r^{1 \prime}\right)
\end{gathered}
$$


in terms of which the constraints read

$$
\Theta_{ \pm}= \pm P_{ \pm} X^{ \pm \prime}+\theta_{ \pm}
$$

where $\theta_{ \pm}$is just the matter part

$$
\theta_{ \pm}=\frac{1}{4}\left(\Pi \pm \varphi^{\prime}\right)^{2}
$$

The gravitational contribution to $\Theta_{ \pm}$has been transformed into $\pm P_{ \pm} X^{ \pm \prime}$, which looks like the momentum density for fields $\left\{P_{ \pm}, X^{ \pm}\right\}$. One sees once again that the gravity portions of the constraint do not give rise to an anomaly: a momentum density commutator possesses no anomaly, see (4.2) - it is present only in the matter-part commutator $\left[\theta_{ \pm}, \theta_{ \pm}\right]$.

With Kuchař [10], we now ask: is it possible to change the theory by adding something to $\Theta_{ \pm}$, so that the modified constraints possess no anomaly? A remarkably simple expression is found to do the job. One verifies that the algebra of $\widetilde{\Theta}_{ \pm}$, defined by

$$
\begin{aligned}
\widetilde{\Theta}_{ \pm} & =\Theta_{ \pm}+\frac{1}{48 \pi}\left(\ln X^{ \pm \prime}\right)^{\prime \prime} \\
& = \pm P_{ \pm} X^{ \pm \prime}+\frac{1}{48 \pi}\left(\frac{X^{ \pm \prime \prime \prime}}{X^{ \pm \prime}}-\left(\frac{X^{ \pm \prime \prime}}{X^{ \pm \prime}}\right)^{2}\right)+: \theta_{ \pm}:
\end{aligned}
$$

possesses no anomaly. Here, $: \theta_{ \pm}:$is normal-ordered with respect to the Fock vacuum defined in (4.7).

[Actually Kuchař's approach [10] is different: he normal orders $\theta_{ \pm}$with respect to a Gaussian (Fock) vacuum with covariance depending on $X^{ \pm}$; this changes the anomaly in the matter commutator $\left[\theta_{ \pm}, \theta_{ \pm}\right]$to an $X^{ \pm}$-dependent expression. He then adds a further $X^{ \pm}$ dependent term to $\Theta_{ \pm}$, whose effect is to cancel the modified anomaly. In our approach, we remain with the conventionally ordered $\theta_{ \pm}$and the conventional anomaly, and find that the relatively simple addition in (4.16) is sufficient. Moreover, (4.16) has a natural interpretation, see below.]

With $\widetilde{\Theta}_{ \pm}$there is no obstruction, and we are instructed to solve

$$
\widetilde{\Theta}_{ \pm}|\psi\rangle=0
$$

or equivalently, in the Schrödinger representation 


$$
\left(\frac{1}{i} \frac{\delta}{\delta X^{ \pm}} \pm \frac{1}{48 \pi X^{ \pm \prime}}\left(\ln X^{ \pm \prime}\right)^{\prime \prime} \pm \frac{1}{X^{ \pm \prime}} \theta_{ \pm}\right) \Psi=0 .
$$

One may say [10,22] that the anomaly is removed by introducing functional U(1) connections

$$
\mathcal{A}_{ \pm}\left(X^{ \pm}\right)= \pm \frac{1}{48 \pi X^{ \pm \prime}}\left(\ln X^{ \pm \prime}\right)^{\prime \prime}
$$

with curvature $\delta \mathcal{A}_{ \pm}\left(X^{ \pm}(\tilde{\sigma})\right) / \delta X^{ \pm}(\sigma)-\delta \mathcal{A}_{ \pm}\left(X^{ \pm}(\sigma)\right) / \delta X^{ \pm}(\tilde{\sigma})$. In the modified constraint $\widetilde{\Theta}_{ \pm}$, one still observes that there is no mixing between gravitational variables $\left\{P_{ \pm}, X^{ \pm}\right\}$and matter variables $\{\Pi, \varphi\}$. But the modified gravitational contribution is no longer quadratic — indeed it is non-polynomial — and we have no idea how to solve (4.17) 23].

The addition that we have made can be related to structures that have already appeared in the literature in descriptions of two-dimensional matter fields interacting with external, c-number gravity [1]. We now explain this.

Observe that our modified action reads

$$
\begin{aligned}
\widetilde{I}=\int & d^{2} x\left\{P_{+} \dot{X}^{+}+P_{-} \dot{X}^{-}+\Pi \dot{\varphi}\right. \\
& -\lambda^{+}\left(P_{+} X^{+\prime}+\frac{1}{48 \pi}\left(\ln X^{+\prime}\right)^{\prime \prime}+\theta_{+}\right) \\
& \left.-\lambda^{-}\left(-P_{-} X^{-\prime}+\frac{1}{48 \pi}\left(\ln X^{-\prime}\right)^{\prime \prime}+\theta_{-}\right)\right\} .
\end{aligned}
$$

Eliminating $P_{ \pm}$evaluates $\lambda^{ \pm}$as $\pm \dot{X}^{ \pm} / X^{ \pm \prime}$ and leaves [24]

$$
\begin{aligned}
\widetilde{I} & =I_{0}+\Delta I \\
I_{0} & =\int d^{2} x\left(\Pi \dot{\varphi}-\frac{\dot{X}^{+}}{X^{+\prime}} \theta_{+}+\frac{\dot{X}^{-}}{X^{-\prime}} \theta_{-}\right) \\
\Delta I & =\frac{1}{48 \pi} \int d^{2} x\left(-\frac{\dot{X}^{+}}{X^{+\prime}}\left(\ln X^{+\prime}\right)^{\prime \prime}+\frac{\dot{X}^{-}}{X^{-\prime}}\left(\ln X^{-\prime}\right)^{\prime \prime}\right) .
\end{aligned}
$$

$I_{0}$ is recognized to be just the matter action (1.4), written in first order from (so that metric tensor components enter only as Lagrange multipliers) with $g_{\mu \nu}$ parametrized as

$$
g_{\mu \nu}=e^{\chi} \partial_{\mu} X^{a} \partial_{\nu} X^{b} h_{a b},
$$

and

$$
\theta_{ \pm}=\frac{1}{4}\left(\sqrt{-g} g^{0 \mu} \partial_{\mu} \varphi \pm \varphi^{\prime}\right)^{2}=\frac{1}{4}\left(\Pi \pm \varphi^{\prime}\right)^{2}
$$


It is well known that the energy momentum tensor $\theta_{\mu \nu}$ for the matter variables of the theory described by $I_{0}$

$$
\theta_{\mu \nu}=\frac{2}{\sqrt{-g}} \frac{\delta I_{0}}{\delta g^{\mu \nu}},
$$

exhibits a quantal anomaly, which may be viewed as a diffeomorphism anomaly or a trace anomaly. Usually this is described by computing the matrix elements of $\theta_{\mu \nu}$ in the presence of a background $g_{\mu \nu},\left\langle\theta_{\mu \nu}\right\rangle_{g}$, and examining the divergence and trace of $\left\langle\theta_{\mu \nu}\right\rangle_{g}$ [25]. Alternatively one may functionally integrate the matter variables, obtain a nonlocal effective gravitational action, $I_{\text {effective }}(g)$, which is functional of $g_{\mu \nu}$, and study its diffeomorphism and Weyl invariance properties [3]. Evidently $\left\langle\theta_{\mu \nu}\right\rangle_{g}=(2 / \sqrt{-g})\left(\delta I_{\text {effective }}(g) / \delta g^{\mu \nu}\right)$.

Still another approach is the following [11]. Form $\theta_{\mu \nu}$ as in (4.25) and express it in terms of $\theta_{+}$and $\theta_{-}$in (4.24), viewing these to be quantum operators satisfying the commutator algebra 4.12

$$
\begin{aligned}
& \theta_{++}=\frac{1}{2}\left(\lambda^{+}+1\right)^{2} \theta_{+}+\frac{1}{2}\left(\lambda^{-}-1\right)^{2} \theta_{-}, \\
& \theta_{--}=\frac{1}{2}\left(\lambda^{+}-1\right)^{2} \theta_{+}+\frac{1}{2}\left(\lambda^{-}+1\right)^{2} \theta_{-}, \\
& \theta_{+-}=\frac{1}{2}\left(\left(\lambda^{+}\right)^{2}-1\right) \theta_{+}+\frac{1}{2}\left(\left(\lambda^{-}\right)^{2}-1\right) \theta_{-} .
\end{aligned}
$$

Here $\lambda^{ \pm}$is the c-numbers $\lambda^{ \pm}=\left(-\sqrt{-g} \pm g_{01}\right) / g_{11}$. It follows that the operator $\theta_{\mu \nu}$ is traceless. Next compute the covariant divergence of $\theta_{\mu \nu}$, where time derivatives are calculated as commutators with the Hamiltonian,

$$
\begin{aligned}
H & =\int d \sigma\left(\lambda^{+} \theta_{+}+\lambda^{-} \theta_{-}\right), \\
\dot{\theta}_{ \pm} & =i\left[H, \theta_{ \pm}\right]
\end{aligned}
$$

and the quantum anomaly in the $\left[\theta_{ \pm}, \theta_{ \pm}\right]$commutator is taken into account. One finds non-vanishing c-numbers for the divergence

$$
\begin{aligned}
& D_{\mu} \theta_{+}^{\mu}=-\frac{1}{24 \pi \sqrt{-g}} \frac{1}{\sqrt{2}}\left(\left(\lambda^{+}+1\right) \lambda^{+\prime \prime \prime}-\left(\lambda^{-}-1\right) \lambda^{-\prime \prime \prime}\right), \\
& D_{\mu} \theta_{-}^{\mu}=-\frac{1}{24 \pi \sqrt{-g}} \frac{1}{\sqrt{2}}\left(\left(\lambda^{+}-1\right) \lambda^{+\prime \prime \prime}-\left(\lambda^{-}+1\right) \lambda^{-\prime \prime \prime}\right) .
\end{aligned}
$$

Finally one asks whether there is a counter action $\Delta I$, constructed solely from $g_{\mu \nu}$, which when summed with $I_{0}$ 


$$
\widetilde{I}=I_{0}+\Delta I
$$

produces a traceless and conserved energy-momentum tensor

$$
\begin{aligned}
\widetilde{\theta}_{\mu \nu} & =\frac{2}{\sqrt{-g}} \frac{\delta \widetilde{I}}{\delta g^{\mu \nu}}, \\
D_{\mu} \widetilde{\theta}_{\nu}^{\mu} & =0 .
\end{aligned}
$$

The answer is that $\Delta I$ in (4.22) does the job, with $\lambda^{ \pm}= \pm \dot{X}^{ \pm} / X^{ \pm \prime}$, because the c-number covariant divergence of $(2 / \sqrt{-g}) \delta \Delta I / \delta g^{\mu \nu}$ cancels the right sides of (4.29).

We conclude this Section with various observations.

1. Even though $I_{0}$ in (4.21) is a matter action like (1.4), but presented in first-order form, the dynamical equations that follow from (4.21) are not identical to those of (1.4). As was seen, our transformations on the CGHS theory result in the parameterization (4.23) for the effective metric tensor, which occurs in the final matter action (4.21). When the metric tensor is varied as a whole, which one can do in (1.4), one gets the (classical) equation that $\theta_{\mu \nu}$ must vanish. On the other hand, when $g_{\mu \nu}$ is given by (4.23), one can only vary $\chi$ and $X^{a}$; this gives weaker conditions: $\theta^{\mu}{ }_{\mu}=0$ and $\theta^{\mu \nu}\left(D_{\mu} V_{\nu}^{a}+D_{\nu} V_{\mu}^{a}-g_{\mu \nu} D^{\alpha} V_{\alpha}^{a}\right)=0$, where $V_{\mu}^{a}=(\exp \chi) \partial_{\mu} X^{a}$ and (classical) conservation of $\theta^{\mu \nu}$ has been used.

2. The consistent theory that we have constructed is diffeomorphism invariant because the commutator anomaly has been removed, and also it is Weyl invariant because the compensating term $\Delta I$ does not involve the conformal factor of the metric. This is possible because the counter term, which is a local expression in terms of $X^{ \pm}$that are the natural variables of the (transformed) CGHS theory, would be nonlocal if expressed in terms of a metric tensor.

3. There is another route to the conclusion that elimination of the gravitational variables in (1.6), (1.7) results in an action for matter coupled to a metric. Observe that $\mathcal{L}$ in (1.6) is equivalent to a second order Lagrangian for three fields $\Phi^{A}=\left(r^{a}, \varphi\right)$, with the index $A$ being governed by the metric tensor $\operatorname{diag}(1,-1,1)$ 


$$
\mathcal{L}_{g+m} \rightarrow \mathcal{L}_{\text {equivalent }}=\frac{\sqrt{-\mathbf{g}}}{2} \mathbf{g}^{\mu \nu} \partial_{\mu} \Phi^{A} \partial_{\nu} \Phi_{A}
$$

Here $\mathbf{g}^{\mu \nu}$ is a fictitious metric tensor, unrelated to the gravitational variables of the theory, but producing the Lagrange multipliers $u$ and $v$ in (1.6)

$$
u=\frac{-\sqrt{-\mathbf{g}}}{\mathbf{g}_{11}}, \quad v=-\frac{\mathbf{g}_{01}}{\mathbf{g}_{11}}
$$

Consequently, forming the equivalent action, $I_{\text {equivalent }}=\int d^{2} x \mathcal{L}_{\text {equivalent }}$, and functionally integrating $e^{i I_{\text {equivalent }}}$ over the first two fields, i.e. over $r^{a}$, produces unity, because of the indefinite metric: integration of $r^{0}$ involves only the integrand $e^{\frac{i}{2} \int d^{2} x \sqrt{-\mathbf{g}} \mathbf{g}^{\mu \nu} \partial_{\mu} r^{0} \partial_{\nu} r^{0}}$ and yields $e^{i I_{\text {effective }}(\mathbf{g})}$, integration of $r^{1}$ involves the integrand $e^{-\frac{i}{2} \int d^{2} x \sqrt{-\mathbf{g}} \mathbf{g}^{\mu \nu} \partial_{\mu} r^{1} \partial_{\nu} r^{1}}$ and yields $e^{-i I_{\text {effective }}(\mathbf{g})}$. Thus integration over the two gravitational variables gives unity and leaves only the matter term $e^{\frac{i}{2} \int d^{2} x \sqrt{-\mathbf{g}} \mathbf{g}^{\mu \nu} \partial_{\mu} \varphi \partial_{\nu} \varphi}$.

4. Observe that our addition $\Delta I$ in (4.22) is composed of two terms: one involving $X^{+}$, the other $X^{-}$. One may verify that each term is proportional to the induced Polyakov action [26] $\int \sqrt{-g} R\left(D^{\mu} D_{\mu}\right)^{-1} R$, in light-cone gauge [27]. [Summation formulas for two Polyakov actions can be given, but they involve a cross term [28], so that the sum occurring in (4.22) does not appear to be expressible in terms of single Polyakov action.]

5. Finally we remark that the commutator anomaly in (4.12a) may alternatively be cancelled by an addition to $\Theta_{ \pm}$quite different from (4.16). One easily verifies that $\Theta_{ \pm}+\alpha_{ \pm} P_{ \pm}^{\prime}+\beta_{ \pm} X^{ \pm^{\prime \prime}}$ closes with no triple derivative Schwinger term provided $\alpha_{ \pm} \beta_{ \pm}=\mp(1 / 48 \pi)$. Owing to the presence of $P_{ \pm}^{\prime}$, this modification does not have the interpretation of a functional connection; in fact it is related to familiar "improvements" of the energy-momentum tensor, which will be discussed in Section V1. 


\section{NEGATIVE ENERGY SCALARS AND STATES IN PURE DILATON GRAVITY}

In this Section we consider quantization of a scalar field that enters the Lagrangian with a negative kinetic term [such a field is present in the dilaton gravity Lagrangian, see (1.7)] and show there are two options. In the first option, standard in string theory, one works with positive energy states (some of negative norm) and finds a positive central term, which therefore adds to the one coming from a conventionally signed Lagrangian, and thus even pure dilaton gravity, without matter, possesses an obstruction in its constraint algebra, with resulting center of $(+2)$.

However, we can adopt a second option, where one works with positive norm states carrying negative energy. This leads to a negative central term, which cancels the one coming from a conventionally signed Lagrangian, thereby removing the obstruction in the pure dilaton gravity constraint algebra. This is the choice made in Section IV. [Of course, when states are taken to be explicit, Schrödinger representation functionals of the canonical (coordinate) fields, negative norms cannot be achieved, and only the second option is available, as in Section IV.] In this way we regain the quantization scheme of Refs. [6, 9, 19,21, which is different from the usual string theory approach. There are two physical states and we give their explicit form in oscillator language. These results can be interpreted as providing a quantization for a string on two-dimensional Minkowski space, where all Virasoro constraints are satisfied, without introducing ghosts.

\section{A. Indefinite sign quadratic forms and central terms}

Let us consider a free scalar field $\xi(t, \sigma)$ in two space-time dimensions $(t, \sigma)$. To make rapid contact with string theory results, the spatial coordinate $\sigma$ is taken on a circle: $\sigma \in$ $[0,2 \pi]$, but this does not alter significantly the infinite-line results of the previous Sections. Since we are interested in comparing scalars with positive and negative kinetic terms, we let $\xi_{+}\left(\xi_{-}\right)$denote the former (latter). Henceforth, the $( \pm)$subscripts do not denote lightcone components; rather they signal positive $(+)$ or negative $(-)$ kinetic terms for $\xi_{ \pm}$. In a 
convenient normalization, the Minkowski action $I_{ \pm}^{M}$ for both scalar fields reads

$$
I_{ \pm}^{M}= \pm \frac{1}{8 \pi} \int d t d \sigma\left[\dot{\xi}_{ \pm}^{2}-\xi_{ \pm}^{\prime 2}\right]
$$

No additional interactions are included, because as is seen from equations (4.6), the dynamical problem in our final version of the pure dilaton gravity model is governed by quadratic energy and momentum densities that must annihilate physical states. The fields in (5.1) correspond to the gravitational $r^{a}$ degrees of freedom in (4.6).

As usual in path integral quantization, the weight factor is $\exp \left(i I_{ \pm}^{M}\right)$. The canonical momenta $\Pi_{ \pm}$and Hamiltonians $H_{ \pm}$for this system are readily found:

$$
\begin{gathered}
\Pi_{ \pm}= \pm \frac{1}{4 \pi} \dot{\xi}_{ \pm} \\
H_{ \pm}= \pm \frac{1}{2} \int_{0}^{2 \pi} d \sigma\left(4 \pi \Pi_{ \pm}^{2}+\frac{1}{4 \pi} \xi_{ \pm}^{\prime 2}\right)
\end{gathered}
$$

The basic equal time commutator is

$$
\left[\xi_{ \pm}(\sigma), \Pi_{ \pm}(\tilde{\sigma})\right]=i \delta(\sigma-\tilde{\sigma})
$$

(A common time argument is suppressed.) The Hamiltonian equations are solved by a set of oscillator expansions.

$$
\begin{aligned}
\xi_{ \pm}(t, \sigma) & =x_{ \pm}+2 t p_{ \pm}+i \sum_{n \neq 0} \frac{1}{n}\left[\alpha_{n}^{ \pm} e^{-i n(t-\sigma)}+\bar{\alpha}_{n}^{ \pm} e^{-i n(t+\sigma)}\right] \\
\pm 2 \pi \Pi_{ \pm}(t, \sigma) & =p_{ \pm}+\frac{1}{2} \sum_{n \neq 0}\left[\alpha_{n}^{ \pm} e^{-i n(t-\sigma)}+\bar{\alpha}_{n}^{ \pm} e^{-i n(t+\sigma)}\right]
\end{aligned}
$$

where hermiticity requires $\left(\alpha_{n}^{ \pm}\right)^{\dagger}=\alpha_{-n}^{ \pm}$, and $\left(\bar{\alpha}_{n}^{ \pm}\right)^{\dagger}=\bar{\alpha}_{-n}^{ \pm}$. The non-vanishing commutators are determined by (5.4)

$$
\left[x_{ \pm}, p_{ \pm}\right]= \pm i, \quad\left[\alpha_{m}^{ \pm}, \alpha_{n}^{ \pm}\right]= \pm m \delta_{m+n, 0}, \quad\left[\bar{\alpha}_{m}^{ \pm}, \bar{\alpha}_{n}^{ \pm}\right]= \pm m \delta_{m+n, 0}
$$

Note that the commutation relations for the oscillators corresponding to the two different scalars differ by a sign.

We define $p_{ \pm}=\alpha_{0}^{ \pm}=\bar{\alpha}_{0}^{ \pm}$, and take the vacuum state $|0\rangle$ to be annihilated by all the operators $\alpha_{n}^{ \pm}$and $\bar{\alpha}_{n}{ }^{ \pm}$with $n \geq 0$ : 


$$
\alpha_{n}^{ \pm}|0\rangle=\bar{\alpha}_{n}{ }^{ \pm}|0\rangle=0, n \geq 0 .
$$

This follows the usual field theory (and string theory) treatment: the vacuum state is annihilated by the oscillators that appear with positive frequencies in the expansion of the field operators, and this choice is made for both scalars. An important consequence follows: the states created by the creation operators have positive energy for both scalars. For scalars with negative kinetic energy, the norm of some states will be negative $\left(e . g \cdot \alpha_{-|n|}^{-}|0\rangle\right)$, and as will be seen below, the vacuum is not a localized wave functional in the Schrödinger representation. Alternatively, another choice of creation and annihilation operators allows for a localized vacuum wave functional and gives different conclusions about the structure of the theory; this will be explained later.

We now continue with the analysis. It follows from (5.5) that at $t=0$

$$
\frac{1}{2}\left(\dot{\xi}_{ \pm}-\xi_{ \pm}^{\prime}\right)=\sum_{n} \alpha_{n}^{ \pm} e^{i n \sigma}, \quad \frac{1}{2}\left(\dot{\xi}_{ \pm}+\xi_{ \pm}^{\prime}\right)=\sum_{n} \bar{\alpha}_{n}^{ \pm} e^{-i n \sigma} .
$$

Furthermore, also at $t=0$

$$
\begin{aligned}
\pm \frac{1}{4}\left(\dot{\xi}_{ \pm}^{2}+{\xi^{\prime}}_{ \pm}^{2}\right) & =\sum_{p}\left(e^{-i p \sigma} \bar{L}_{p}^{ \pm}+e^{i p \sigma} L_{p}^{ \pm}\right) \\
\pm \frac{1}{2} \dot{\xi}_{ \pm} \xi_{ \pm}^{\prime} & =\sum_{p}\left(e^{-i p \sigma} \bar{L}_{p}^{ \pm}-e^{i p \sigma} L_{p}^{ \pm}\right)
\end{aligned}
$$

where all operators are normal ordered with respect to the vacuum defined in (5.7), and

$$
\begin{aligned}
L_{p}^{ \pm} & = \pm \frac{1}{2} \sum_{n}: \alpha_{p+n}^{ \pm} \alpha_{-n}^{ \pm}: \\
\bar{L}_{p}^{ \pm} & = \pm \frac{1}{2} \sum_{n}: \bar{\alpha}_{p+n}^{ \pm} \bar{\alpha}_{-n}^{ \pm}:
\end{aligned}
$$

These are the Virasoro operators. From (5.2), (5.9) and (5.10), we see that the Hamiltonians are given by

$$
H_{ \pm}=L_{0}^{ \pm}+\bar{L}_{0}^{ \pm}= \pm\left(p_{ \pm}^{2}+\sum_{n=1}^{\infty}\left(\alpha_{-n}^{ \pm} \alpha_{n}^{ \pm}+\bar{\alpha}_{-n}^{ \pm} \bar{\alpha}_{n}^{ \pm}\right)\right)
$$

and the reader can verify that the creation operators indeed increase the energy both for the case of $\xi_{+}$and $\xi_{-}$. The Virasoro operators obey the following commutation relations

$$
\left[L_{m}^{ \pm}, L_{n}^{ \pm}\right]=(m-n) L_{m+n}^{ \pm}+\frac{c_{ \pm}}{12}\left(m^{3}-m\right) \delta_{m+n, 0} .
$$


with the $\bar{L}_{m}^{ \pm}$'s satisfying exactly the same commutators. The central charge $c_{ \pm}$is easily obtained from

$$
\frac{c_{ \pm}}{2}=\left\{\begin{array}{l}
\left\langle 0\left|\left[L_{2}^{ \pm}, L_{-2}^{ \pm}\right]\right| 0\right\rangle=\left\langle 0\left|L_{2}^{ \pm} L_{-2}^{ \pm}\right| 0\right\rangle=\frac{1}{4}\left\langle 0\left|\alpha_{1}^{ \pm} \alpha_{1}^{ \pm} \alpha_{-1}^{ \pm} \alpha_{-1}^{ \pm}\right| 0\right\rangle \\
\left\langle 0\left|\left[\bar{L}_{2}^{ \pm}, \bar{L}_{-2}^{ \pm}\right]\right| 0\right\rangle=\left\langle 0\left|\bar{L}_{2}^{ \pm} \bar{L}_{-2}^{ \pm}\right| 0\right\rangle=\frac{1}{4}\left\langle 0\left|\bar{\alpha}_{1}^{ \pm} \bar{\alpha}_{1}^{ \pm} \bar{\alpha}_{-1}^{ \pm} \bar{\alpha}_{-1}^{ \pm}\right| 0\right\rangle
\end{array}\right\} .
$$

We now use the commutation relations (5.6), and find that $c_{ \pm}=1$ for the scalars with either positive or negative kinetic term. While the commutation relations for the respective oscillators differ by a sign, they must be used twice, and therefore give the same sign in both cases.

This result can also be presented in the context of the $[\mathcal{E}, \mathcal{P}]$ commutator. The energymomentum tensor for the scalars $\xi_{ \pm}$is given by

$$
T_{\mu \nu}^{ \pm}= \pm \frac{1}{2}\left(\partial_{\mu} \xi_{ \pm} \partial_{\nu} \xi_{ \pm}-\frac{1}{2} \eta_{\mu \nu}\left(\partial \xi_{ \pm}\right)^{2}\right)
$$

where $\eta_{\mu \nu}=\operatorname{diag}(1,-1)$. It follows that

$$
\begin{aligned}
& \mathcal{E}^{ \pm}(\sigma) \equiv \frac{1}{2 \pi} T_{00}^{ \pm}(\sigma)= \pm \frac{1}{8 \pi}\left(\dot{\xi}_{ \pm}^{2}+\xi_{ \pm}^{\prime 2}\right) \equiv \frac{1}{2 \pi} \sum_{n} e^{-i n \sigma} \mathcal{E}_{n}^{ \pm} \\
& \mathcal{P}^{ \pm}(\sigma) \equiv-\frac{1}{2 \pi} T_{01}^{ \pm}(\sigma)=\mp \frac{1}{4 \pi} \dot{\xi}_{ \pm} \xi_{ \pm}^{\prime} \equiv-\frac{1}{2 \pi} \sum_{n} e^{-i n \sigma} \mathcal{P}_{n}^{ \pm}
\end{aligned}
$$

Direct comparison with Eq. (5.9) gives

$$
\mathcal{E}_{n}^{ \pm}=\bar{L}_{n}^{ \pm}+L_{-n}^{ \pm}, \quad \mathcal{P}_{n}^{ \pm}=\bar{L}_{n}^{ \pm}-L_{-n}^{ \pm}
$$

It is now a simple matter to use (5.12) (and its analog for the $\bar{L}_{n}^{ \pm}$'s) to find

$$
\left[\mathcal{E}_{m}^{ \pm}, \mathcal{P}_{n}^{ \pm}\right]=(m-n) \mathcal{E}_{m+n}^{ \pm}+\frac{c_{ \pm}}{6}\left(m^{3}-m\right) \delta_{m+n, 0}
$$

Since we have shown that $c_{ \pm}=1$, it follows from the above equation that there is a central term in the $\left[\mathcal{E}_{m}^{+}+\mathcal{E}_{m}^{-}, \mathcal{P}_{n}^{+}+\mathcal{P}_{n}^{-}\right]$commutator. From the definitions (5.15) we conclude that, with the quantization scheme described in this subSection, there is a central term in the $\left[\mathcal{E}^{+}(\sigma)+\mathcal{E}^{-}(\sigma), \mathcal{P}^{+}(\sigma)+\mathcal{P}^{-}(\sigma)\right]$ commutator of the total energy and total momentum densities. Thus the combined system of a positive norm and a negative norm scalar exhibits a central term in the relevant commutator, so that the total $\mathcal{E}$ and $\mathcal{P}$ operators cannot annihilate a state. 


\section{B. Another choice}

Since the Lagrangian with negative kinetic term gives rise to a Hamiltonian that appears negative (in the above treatment positive energies are nevertheless achieved at the expense of negative-norm states), one could say that the annihilation operator corresponds to a negative frequency oscillator in the expansion of field operators. Thus, in contrast to the usual conformal field theory choice (5.7), we can take

$$
\begin{aligned}
& \alpha_{n}^{+}|0\rangle=\bar{\alpha}_{n}{ }^{+}|0\rangle=0, n \geq 0, \\
& \alpha_{n}^{-}|0\rangle=\bar{\alpha}_{n}{ }^{-}|0\rangle=0, n \leq 0,
\end{aligned}
$$

and now there are no negative norm states.

A further argument in favor of (5.18) can be made. Note that the positive frequency modes are projected by

$$
\int_{0}^{2 \pi} d \sigma e^{-i \sigma n}\left( \pm \Pi_{ \pm}(t, \sigma)-\frac{i|n|}{4 \pi} \xi_{ \pm}(t, \sigma)\right)= \begin{cases}\alpha_{n}^{ \pm} e^{-i n t} & n>0 \\ \bar{\alpha}_{|n|}^{ \pm} e^{-i|n| t} & n<0\end{cases}
$$

This may also be presented with the aid of the positive kernel

$$
\begin{gathered}
\omega(\sigma, \tilde{\sigma})=\frac{1}{8 \pi^{2}} \sum_{n} e^{i n(\sigma-\tilde{\sigma})}|n|, \\
\int_{0}^{2 \pi} d \sigma e^{-i \sigma n}\left( \pm \Pi_{ \pm}(0, \sigma)-i \int_{0}^{2 \pi} d \tilde{\sigma} \omega(\sigma, \tilde{\sigma}) \xi_{ \pm}(0, \tilde{\sigma})\right)= \begin{cases}\alpha_{n}^{ \pm} & n>0, \\
\bar{\alpha}_{|n|}^{ \pm} & n<0 .\end{cases}
\end{gathered}
$$

When these modes are taken to annihilate the vacuum, as in conformal theory [see (5.7) ], we have in the Schrödinger representation

$$
\left\{ \pm \frac{\delta}{\delta \xi_{ \pm}(\sigma)}+\int_{0}^{2 \pi} d \tilde{\sigma} \omega(\sigma, \tilde{\sigma}) \xi_{ \pm}(\tilde{\sigma})\right\} \Psi_{ \pm}^{0}\left(\xi_{ \pm}\right)=0
$$

where $\Psi_{ \pm}^{0}$ is the vacuum functional depending on $\xi_{ \pm}(\sigma) \equiv \xi_{ \pm}(0, \sigma)$. The unique (up to normalization) solution is

$$
\Psi_{ \pm}^{0}\left(\xi_{ \pm}\right)=\exp \left(\mp \frac{1}{2} \int \xi_{ \pm} \omega \xi_{ \pm}\right)
$$

We see that for $\xi_{-}$, whose kinetic term is negative, the functional grows inadmissibly as a quadratic exponential, since the kernel is positive. On the contrary if in this case the 
modes annihilating the vacuum are taken with negative frequency, one obtains an acceptable Gaussian, as in the usual case with positive kinetic term [see (4.7)].

With the choice (5.18) one readily verifies that states created with the positively moded oscillators have positive norms, but negative energies. It also follows that the central term $c_{-}$corresponding to the scalar with negative kinetic term, quantized in this way, is given by $c_{-}=-1$. Indeed, the computation in (5.13) changes by a sign since this time $L_{-2}$ annihilates the vacuum [29].

With the ordering prescription we are considering now, there is no central term in $\left[\mathcal{E}^{+}(\sigma)+\right.$ $\left.\mathcal{E}^{-}(\sigma), \mathcal{P}^{+}(\tilde{\sigma})+\mathcal{P}^{-}(\tilde{\sigma})\right]$, so that the total $\mathcal{E}$ and $\mathcal{P}$ operators can annihilate a state. The relevant states, in the Schrödinger representation, read $\exp \left( \pm \frac{i}{4 \pi} \int d \sigma \xi_{+} \xi_{-}^{\prime}\right)$ [compare (4.5)], and we seek their Fock space equivalents to verify explicitly that they are annihilated by all the Virasoro conditions. There is a well-defined procedure to go from a wave functional to its corresponding state, and coherent state methods are most efficient. Since these methods are familiar, we just give the result, which follows after a certain amount of simple computation. The relation of the above state to the Fock vacuum is given by [compare (4.8)]

$$
| \pm\rangle=\left\{\prod_{n=1}^{\infty} 2 \cdot \exp \left( \pm \frac{1}{n}\left[\alpha_{-n}^{+} \alpha_{n}^{-}-\bar{\alpha}_{-n}^{+} \bar{\alpha}_{n}^{-}\right]\right)\right\}|0\rangle
$$

Recall that for the positive signature scalar the negatively moded oscillators are creation operators and for the negative signature scalar the positively moded oscillators are creation operators. It follows that all the oscillators appearing in the above exponential are creation operators, as they should be for a normal ordered representation of the state. It is interesting to note that the straightforward transcription from the Schrödinger representation gives a state with the factor of two shown above, which prevents the state from being a linear superposition with finite coefficients of Fock space states (states built with a finite number of oscillators acting on the vacuum). We can drop this factor and concentrate on the nontrivial part of the states

$$
\left|\Psi_{ \pm}\right\rangle=\exp \left( \pm \sum_{n=1}^{\infty} \frac{1}{n}\left[\alpha_{-n}^{+} \alpha_{n}^{-}-\bar{\alpha}_{-n}^{+} \bar{\alpha}_{n}^{-}\right]\right)|0\rangle
$$

The Virasoro conditions or physical state conditions demand that the operators $\mathcal{E}_{n}^{ \pm}$and 
$\mathcal{P}_{n}^{ \pm}$given in (5.16) must annihilate $\left|\Psi_{ \pm}\right\rangle$for all values of $n$. By taking suitable linear combinations of these constraints they can be put in the form $\mathcal{L}_{m}\left|\Psi_{ \pm}\right\rangle=\overline{\mathcal{L}}_{m}\left|\Psi_{ \pm}\right\rangle=0$ for all $m$ where the operators

$$
\mathcal{L}_{m} \equiv L_{m}^{+}+L_{m}^{-}, \quad \overline{\mathcal{L}}_{m} \equiv \bar{L}_{m}^{+}+\bar{L}_{m}^{-}
$$

are the total Virasoro operators of the system, and the relevant oscillator expansions are given in (5.10). Because holomorphic and antiholomorphic sectors of the constraints decouple, it is sufficient to verify that

$$
\mathcal{L}_{m} e^{ \pm \Omega}|0\rangle=0, \quad \text { where } \quad \Omega=\sum_{n=1}^{\infty} \frac{1}{n} \alpha_{-n}^{+} \alpha_{n}^{-}
$$

Since the Virasoro operators have at most two annihilators and $\Omega$ has two creators, multiple commutators with more than two $\Omega$ 's must vanish. It is therefore enough to verify that

$$
\left(\mathcal{L}_{m}+\left[\mathcal{L}_{m}, \pm \Omega\right]+\frac{1}{2}\left[\left[\mathcal{L}_{m}, \Omega\right], \Omega\right]\right)|0\rangle=0
$$

One readily checks that the above equation is satisfied since $\left[\mathcal{L}_{m}, \pm \Omega\right]|0\rangle=0$, and the other two terms cancel each other. As a consequence (5.27) holds, and the states $\left|\Psi_{ \pm}\right\rangle$satisfy all the Virasoro constraints.

This result can be interpreted as providing physical states in a quantization of a string on two-dimensional Minkowski space without introducing ghosts. To be sure, the state space for this string, being two-fold, is rather small. Nevertheless, it is interesting that all Virasoro constraints can be satisfied, and of course in the gravitational context, the states give an adequate description of pure dilaton gravity on a line [6,9,19,21]. A similar construction can also be carried out for a string in $(d, d)$ dimensional space-time.

A final observation is in order. Since the constraints decouple the holomorphic and antiholomorphic sector, we actually find four states satisfying the constraints: the extra two corresponding to the remaining sign combinations that can be constructed in (5.25). Nevertheless, one can show that such states do not have associated wave functionals; the procedure for obtaining wave functionals from states does not work due to infinities. We therefore suspect that these other two states are not likely to be relevant. There may be 
other physical states, but we have not found them. (The strategy of multiplying $\left|\Psi_{ \pm}\right\rangle$by an operator that commutes with all Virasoro operators does not give anything new.)

\section{ACHIEVING ZERO CENTER WITHIN THE CONFORMAL APPROACH}

In this Section we go back to the quantization choice for the negative signed scalar field conventional in conformal theory, so that its center is $c_{-}=1$. We use the Euclidean

treatment with $z \equiv \tau+i \sigma, \bar{z} \equiv \tau-i \sigma,\left(\partial \equiv \frac{\partial}{\partial z}, \bar{\partial} \equiv \frac{\partial}{\partial \bar{z}}\right)$ but retain indefinite signature for the two gravitational scalar fields. We discuss "improvements" of the energy-momentum tensor and then show how to achieve constraints without a center.

\section{A. Central terms and improvements}

Let $\xi_{+}$and $\xi_{-}$denote free scalar Euclidean fields with positive and negative kinetic terms respectively. For both scalars we set $\xi_{ \pm}(z, \bar{z})=\xi_{ \pm}(z)+\bar{\xi}_{ \pm}(\bar{z})$, as implied by the equation of motion, and one has

$$
\left\langle\xi_{ \pm}\left(z_{1}\right) \xi_{ \pm}\left(z_{2}\right)\right\rangle=\mp \ln \left(z_{1}-z_{2}\right), \quad\left\langle\bar{\xi}_{ \pm}\left(\bar{z}_{1}\right) \bar{\xi}_{ \pm}\left(\bar{z}_{2}\right)\right\rangle=\mp \ln \left(\bar{z}_{1}-\bar{z}_{2}\right)
$$

We shall now discuss the standard improvement terms that can change the central charge of a two-dimensional scalar field. Consider the holomorphic component of the energymomentum tensor, improved by a linear term

$$
T_{ \pm}(z)=\mp \frac{1}{2} \partial \xi_{ \pm}(z) \partial \xi_{ \pm}(z)+\frac{Q_{ \pm}}{2} \partial^{2} \xi_{ \pm}(z)
$$

where we take the constants $Q_{ \pm}$to be real (in Minkowski space this makes the extra term in the energy-momentum tensor real). A short calculation gives

$$
c_{ \pm}=1 \pm 3 Q_{ \pm}^{2}
$$

Therefore the improvement increases the central charge of the positively signed scalar $\xi_{+}$, and decreases the one of the negatively signed scalar $\xi_{-}$. The constants $Q_{+}$and $Q_{-}$are arbitrary. Choosing them allows setting the total central charge to any desired value, and 
various choices will be made below. For definiteness we shall take the choice where $Q_{+}$or $Q_{-}$vanishes, and the other is adjusted to fix the total central charge.

For later reference we note that when the (holomorphic) energy-momentum tensor for a free Bose field $\chi(z, \bar{z})=\chi(z)+\bar{\chi}(\bar{z})$ takes the form

$$
T(z)=-\frac{1}{2} \partial \chi(z) \partial \chi(z)+\frac{Q}{2} \partial^{2} \chi(z)
$$

then the local operators $e^{w \chi(z)}$ have dimension

$$
\operatorname{dim}\left(e^{w \chi(z)}\right)=\frac{Q^{2}}{8}-\frac{1}{2}\left(w-\frac{Q}{2}\right)^{2}
$$

Moreover, since the translation current $i \partial \chi$ is no longer a tensor when $Q \neq 0$, the stateoperator correspondence for momentum eigenstates is not the standard one [30].

$$
|p\rangle \Longleftrightarrow \exp \left(i p \chi(z)+\frac{Q}{2} \chi(z)\right)
$$

It follows from the last two equations that

$$
L_{0}|p\rangle=\left(\frac{Q^{2}}{8}+\frac{p^{2}}{2}\right)|p\rangle
$$

\section{B. Achieving zero center}

When we quantize pure dilaton gravity (without matter but with cosmological term) in the way conventional for conformal theory, we obtain a system with central charge $c=2$, and there is an obstruction. The (holomorphic) energy-momentum tensor reads

$$
T=-\frac{1}{2} \partial \xi_{+} \partial \xi_{+}+\frac{1}{2} \partial \xi_{-} \partial \xi_{-}
$$

We improve the negative norm scalar to $c_{-}=-1$ by taking $Q_{-}=\sqrt{2 / 3}$. Thus

$$
\widetilde{T}=-\frac{1}{2} \partial \xi_{+} \partial \xi_{+}+\frac{1}{2} \partial \xi_{-} \partial \xi_{-}+\frac{1}{\sqrt{6}} \partial^{2} \xi_{-},
$$

defines constraints with no obstruction. There is no guarantee, however, that there are interesting states satisfying the constraints. We suspect that the physical states, if any, are

not finite linear superpositions of Fock space states, but rather infinite linear superpositions 
of such states [as is the case for pure dilaton gravity, quantized so that obstructions cancel, see $(5.25)]$.

Let us now include one free matter field, $\varphi(z, \bar{z})=\varphi(z)+\bar{\varphi}(\bar{z})$. The relevant energymomentum tensor is then

$$
T=-\frac{1}{2} \partial \xi_{+} \partial \xi_{+} \quad+\frac{1}{2} \partial \xi_{-} \partial \xi_{-}-\frac{1}{2} \partial \varphi \partial \varphi
$$

This produces a central term $(+3)$ and the resulting constraints cannot be consistently imposed. Again, we improve in the negative norm scalar sector to lower the central charge of that scalar, and get zero for the total center. Taking $Q_{-}=1$ we obtain

$$
\widetilde{T}=-\frac{1}{2} \partial \xi_{+} \partial \xi_{+} \quad+\frac{1}{2} \partial \xi_{-} \partial \xi_{-}+\frac{1}{2} \partial^{2} \xi_{-}-\frac{1}{2} \partial \varphi \partial \varphi
$$

This energy-momentum tensor presents no obstruction to quantization, but again, it remains to be seen whether there are interesting physical states.

Finally we note that in the presence of matter, with gravity quantized as in Section $\mathbb{I V}$, where the negative signed gravitational scalar cancels the center of the positive signed gravitational scalar, there remains the center $c=1$ from the matter field. This may be removed by improving the energy-momentum tensor of the negatively signed scalar as is indicated in point $(5)$ at the end of Section $\left[\mathrm{IV}\right.$. In the present formalism this corresponds to $Q_{-}=\sqrt{2 / 3}$.

\section{BRST QUANTIZATION OF THE DILATON GRAVITY THEORY}

In this Section we consider the quantization of dilaton gravity by the covariant (BRST) method. Conformal field theory conventions are followed for the negative-signed scalar, so that $c_{-}=1$. To begin one must add the reparameterization ghost and antighost, as is justified by noting that the original action is invariant under two-dimensional diffeomorphisms, and BRST quantization requires that the two-dimensional Lagrangian be supplemented by gauge fixing and ghost/antighost terms. Upon selecting the conformal gauge, the Weyl fac-

tor of the two-dimensional metric (Liouville field) together with the dilaton field comprise the two gravitational variables. 
In two-dimensional gravity-matter theories, consistent quantization follows when the combined gravity, matter and ghost degrees of freedom define a conformal field theory with vanishing total central charge [31]. Since the ghost conformal field theory gives a contribution of $(-26)$ to the central charge, the gravity and matter degrees of freedom must give a total central charge of $(+26)$ and this will require changing the central charge by adding a background charge to the energy-momentum tensor of the positively-signed scalar.

This Section has three parts. In the first and second parts, we set up the formalism for pure dilaton gravity and for matter-coupled dilaton gravity respectively. In the third part, we discuss physical states and the semi-classical limit.

\section{A. Pure Dilaton Gravity}

In the BRST approach to quantization, we improve the positive signed scalar $\xi_{+}$to reach total central charge $(+26)$ for both scalars. Then the theory is coupled to the ghost system which has total central charge $(-26)$. We therefore need $Q_{+}=2 \sqrt{2}$ leading to $c_{+}=25$, $c_{+}+c_{-}=26$. The relevant (holomorphic) energy-momentum tensor is therefore given as

$$
\widetilde{T}(z)=T_{\operatorname{gr}}(z)+T_{\operatorname{gh}}(z)
$$

where the improved gravity tensor $T_{\operatorname{gr}}(z)$ and the ghost tensor $T_{\mathrm{gh}}(z)$ are

$$
\begin{aligned}
T_{\mathrm{gr}} & =-\frac{1}{2} \partial \xi_{+} \partial \xi_{+}+\sqrt{2} \partial^{2} \xi_{+}+\frac{1}{2} \partial \xi_{-} \partial \xi_{-}, \\
T_{\text {gh }} & =2(\partial c) b+c \partial b
\end{aligned}
$$

Here $c$ and $b$ are the holomorphic ghost and antighost fields respectively, and satisfy $\langle b(z) c(w)\rangle=1 /(z-w)$. The total energy-momentum tensor $\widetilde{T}$ has no central term. In this quantization procedure physical states are annihilated by the BRST operator

$$
\begin{aligned}
Q_{B} & =\oint d z c(z)\left(T_{\operatorname{gr}}(z)+\frac{1}{2} T_{\mathrm{gh}}(z)\right)+\text { antiholomorphic } \\
\left.Q_{B} \mid \text { phys }\right\rangle & =0
\end{aligned}
$$

Physical states satisfy an equivalence relation (cohomology), 


$$
\mid \text { phys }\rangle \equiv \mid \text { phys }\rangle+Q_{B}|\alpha\rangle
$$

Since, on general grounds $\left\{Q_{B}, b\right\}=\widetilde{T}$, it follows that physical states do not have to be annihilated by $\widetilde{T}$. One just has

$$
\left.\left.\widetilde{T} \mid \text { phys }\rangle=\left\{Q_{B}, b\right\} \mid \text { phys }\right\rangle=Q_{B}(b \mid \text { phys }\rangle\right)
$$

namely, $\widetilde{T}$ on physical states must give a BRST trivial state (almost zero!). It follows that the states annihilated by $\widetilde{T}$ do not coincide with the BRST physical states. While the states annihilated by $\widetilde{T}$ are likely to be very few, the spectrum of BRST physical states is quite rich. In some cases, the BRST physical states are in correspondence with the states annihilated by the positive modes of the matter part of the energy momentum tensor, the so-called "matter primaries".

The total energy-momentum tensor in (7.1)-(7.3) coincides with that of the "Gaussian model", a model where a single boson is minimally coupled to pure two-dimensional gravity (frequently called " $c=1$ " model, highlighting the matter contribution to the central term.). The field $\xi_{+}$plays the role of the Weyl factor in the metric tensor (Liouville field), and $\xi_{-}$ plays the role of the single boson. In the Gaussian model, however, this boson has positive kinetic energy, so its contribution to $T_{\mathrm{gr}}$ enters with opposite sign in (7.2).

\section{B. Matter-coupled dilaton gravity}

Let us now include a single matter field, $\varphi$. The relevant energy-momentum tensor is then

$$
T=-\frac{1}{2} \partial \xi_{+} \partial \xi_{+}+\frac{1}{2} \partial \xi_{-} \partial \xi_{-}-\frac{1}{2} \partial \varphi \partial \varphi
$$

In the BRST approach, we improve $\xi_{+}$to get $c_{+}=24$ and introduce the ghosts. This requires $Q_{+}=\sqrt{23 / 3}$

$$
\widehat{T}=-\frac{1}{2} \partial \xi_{+} \partial \xi_{+}+\sqrt{\frac{23}{12}} \partial^{2} \xi_{+}+\frac{1}{2} \partial \xi_{-} \partial \xi_{-}-\frac{1}{2} \partial \varphi \partial \varphi+2(\partial c) b+c \partial b .
$$


Since this energy-momentum tensor has zero central term one can now find physical states within BRST quantization. We shall discuss the physical states of this theory in the next subSection.

Yet another approach would begin by interpreting the starting point for quantization as a system having three scalar fields $\left(\xi_{+}, \xi_{-}, \varphi\right)$ on a curved world-sheet. Quantization would proceed by adding a further gravitational field $\varphi_{g}$ with $c_{g}=23$, and the $(b, c)$ ghost system. Physical states would be defined by the corresponding BRST operator. We shall not discuss this possibility any further.

\section{Physical states and a comparison with the flat space spectrum}

In this subSection we discuss physical states in the BRST quantization of pure dilaton gravity, and matter-coupled dilaton gravity. To understand the pure dilaton gravity case we begin by discussing the Gaussian model, whose physical states [12] are in correspondence with those of pure dilaton gravity. We then examine a question relevant to the semi-classical limit of the BRST quantized matter-coupled dilaton gravity theory. We use the results of Ref. [13 to enumerate the physical states, and show that the spectrum differs significantly from the spectrum of a free massless particle propagating in flat space in the absence of gravity.

When pure two-dimensional gravity (no dilaton) is coupled minimally to a free massless positive signature boson $X$, one obtains the Gaussian model. This model can be viewed in two different ways: as a quantum gravity field theory in two dimensions with very few physical states, or as a string theory whose target space is two dimensional. Both theories arise as different interpretations for the BRST quantization of the Gaussian model.

The BRST quantization of the Gaussian model proceeds by adding the $(b, c)$ ghost system to account for diffeomorphism invariance, and using a positive signature Liouville field $\varphi_{L}$ to represent the Weyl factor of the metric. A background charge $Q_{L}=2 \sqrt{2}$ is included for $\varphi_{L}$, and thus its center is $c_{L}=25$. Together with the field $X, \varphi_{L}$ forms a system of center $c=26$, the right amount to be cancelled by the ghost system. The physical states of such 
system, defined by the BRST cohomology at the relevant ghost number, are well known [12]. Among the physical states, there is one family parametrized by a continuous parameter. It arises from consideration of the state

$$
\left|V\left(p_{X}, p_{L}\right)\right\rangle=c_{1} \bar{c}_{1}\left|p_{X}, p_{L}\right\rangle
$$

where $c_{1}$ and $\bar{c}_{1}$ are oscillator modes of the fields $c(z)$ and $\bar{c}(\bar{z})$, and $\left|p_{X}, p_{L}\right\rangle$ is a vacuum state carrying momenta $p_{X}$ and $p_{L}$ in the matter and Liouville sectors respectively. For such a state, the BRST condition simply requires that $L_{0}\left|V\left(p_{X}, p_{L}\right)\right\rangle=\bar{L}_{0}\left|V\left(p_{X}, p_{L}\right)\right\rangle=0$. Explicitly these conditions give

$$
L_{0}\left|V\left(p_{X}, p_{L}\right)\right\rangle=\left(-1+\frac{p_{X}^{2}}{2}+1+\frac{p_{L}^{2}}{2}\right) c_{1} \bar{c}_{1}\left|p_{X}, p_{L}\right\rangle=0
$$

and exactly the same equation for $\bar{L}_{0}$. In the above equation we have indicated explicitly the contributions to $L_{0}$ coming from the ghost oscillators $(-1)$, from the matter $\left(\frac{1}{2} p_{X}^{2}\right)$, and from the Liouville sector $\left(1+\frac{1}{2} p_{L}^{2}\right)$, as follows from Eq.66.7). Therefore, the physical state conditions require

$$
p_{X}^{2}+p_{L}^{2}=0 \rightarrow p_{X}=p, \quad p_{L}= \pm i p
$$

where $p$ is an unconstrained real parameter, and the physical states are given as

$$
\left|V_{ \pm}(p)\right\rangle=c_{1} \bar{c}_{1}|p, \pm i p\rangle
$$

In addition to this state, parametrized by a continuous variable $p$, one finds states of the type

$$
\left(\alpha_{-m_{1}} \cdots \alpha_{-m_{p}} \varphi_{-k_{1}} \cdots \varphi_{-k_{q}}\right)\left(\bar{\alpha}_{-n_{1}} \cdots \bar{\alpha}_{-n_{s}} \bar{\varphi}_{-l_{1}} \cdots \bar{\varphi}_{-l_{t}}\right) c_{1} \bar{c}_{1}\left|p_{X}, p_{L}\right\rangle
$$

obtained by acting with some number of creation operators of $X\left(\alpha_{-n}\right.$ and $\left.\bar{\alpha}_{-n}\right)$ and $\varphi_{L}$ $\left(\varphi_{-n}\right.$ and $\left.\bar{\varphi}_{-n}\right)$ on the momentum eigenstate $\left|p_{X}, p_{L}\right\rangle$. Here the BRST conditions force $p_{X}$ and $i p_{L}$ to be fixed real numbers. The BRST conditions also fix specific combinations of creation operators. Due to the absence of a continuous parameter in the allowable momenta these are called discrete states. (There are also more complicated discrete states involving ghost oscillators.) 
We can use the BRST quantization of the Gaussian model as a quantization of pure dilaton gravity. The cohomology problem, which determines physical states, is the same for the two models once we identify the two dilaton gravity fields $\xi_{+}$and $\xi_{-}$with $\varphi_{L}$ and $X$ respectively, except for the change of signature needed to replace $\xi_{-}$with $X$. This change is of little relevance. It is possible to use the results of Bouwknegt.et.al. [12] to see that the set of BRST physical states is not altered except for the changes implied by the signaturealtering identification $X \leftrightarrow i \xi_{-}$. Notice that in this way we obtain a set of physical states for pure dilaton gravity which is far richer than the two states gotten by non-BRST quantization methods described in Section $\square$. At the same time the number of states is not large enough to give rise to a propagating field degree of freedom (see below). This is consistent with expectations from a naive degrees of freedom count: pure two-dimensional gravity giving $3-2 \times 2=-1$, and the dilaton counting as +1 , for a total of zero.

[As a digression let us remark that it makes more sense to interpret the BRST-quantized Gaussian model as pure dilaton gravity, rather than as pure gravity (no dilaton) coupled to a massless matter field. The point is that after BRST quantization almost nothing is left of the scalar field - there are too few quantum states. Indeed the set of BRST physical states bears no resemblance to the set of physical states that would be obtained by quantizing a scalar massless field on a flat two-dimensional space without gravity. If the space coordinate is a circle, the physical states of a single two-dimensional free massless boson can be obtained from the expansion of $\xi_{+}$given in (5.5), and are of the form

$$
\left(\alpha_{-m_{1}}\right)^{n_{1}} \cdots\left(\alpha_{-m_{p}}\right)^{n_{p}}\left(\bar{\alpha}_{-l_{1}}\right)^{r_{1}} \cdots\left(\bar{\alpha}_{-l_{q}}\right)^{r_{q}}|p\rangle
$$

where $p \in[-\infty,+\infty]$ is an arbitrary constant labeling the eigenvalue of the $\Pi_{+}$zero mode. These states, arising from quantization on a cylinder, are the analogs of the many-particle states obtained by quantizing on the infinite space-like line. The state with no oscillators may be paired naturally with the state given in (7.11), but the states with oscillators in (7.13) still have a continuous parameter. That parameter is absent in the discrete BRST states (7.12), moreover, the discrete states are anyway too few. (All this is consistent with the situation in the unquantized, classical theory where the equation for the matter field $X$ 
that follows from varying $g_{\mu \nu}$ requires the vanishing of the matter energy-momentum tensor $\theta_{\mu \nu}=\partial_{\mu} X \partial_{\nu} X-\frac{1}{2} g_{\mu \nu} g^{\alpha \beta} \partial_{\alpha} X \partial_{\beta} X$, which in turn means either that $X$ is constant and $g_{\mu \nu}$ undetermined, or $g_{\mu \nu}$ is singular.)]

The BRST-quantized Gaussian model may alternatively be used to define a twodimensional string theory. In this case $\varphi_{L}$ and $X$ are viewed as coordinates for a twodimensional target space, the space-time of the string theory. Following the standard paradigm, to each state of the Gaussian model one assigns a space-time field, and reinterprets the physical state conditions as linearized equations of motion together with gauge conditions for the space-time field. In doing this one must decide whether $\varphi_{L}$ or $X$ is to be analytically continued to represent time. Both choices have been made in the literature. We shall choose $X$, and thus we let $p_{X} \rightarrow i p_{X}$. In our present context this is suggested from the correspondence with dilaton gravity where the negative signature field $\xi_{-}$is identified with $X$. Following the string paradigm, associated to the state (7.11) we have a scalar field $\eta\left(p_{X}, p_{L}\right)$, whose linearized equation of motion requires $-p_{X}^{2}+p_{L}^{2}=0$. This is therefore a massless scalar field, somewhat misleadingly called the "tachyon". This is the only quantum field in the theory. The discrete states lead to space-time "fields" whose linearized equations of motion and gauge conditions are so strong that they eliminate all degrees of freedom except for a single constant: the value of the field at the allowed momenta. These degrees of freedom are parameters of the background space-time. Sigma model studies have shown that the effective field theory limit of this string theory is precisely the two-dimensional dilaton gravity theory indicated in Eq.(1.1) coupled to a single massless field. To summarize the indirect argument: pure dilaton gravity theory, after transformation to quadratic form, is BRST quantized as a Gaussian model; then a string theory is constructed, which in the effective field theoretic limit reproduces a dilaton gravity model coupled to a single massless field.

We can now proceed with the case of dilaton gravity coupled minimally to a single matter field $\varphi$. The energy-momentum tensor relevant for the BRST quantization of this theory was given in Eq.(7.7). In order to compare this system to those in the literature, it is convenient to label $\left(\xi_{-}, \varphi\right) \equiv\left(X^{0}, X^{1}\right)$ as 'matter' fields, and to label $\xi_{+} \equiv \varphi_{L}$ as the Liouville field . 
The energy-momentum tensor then reads (with $Q=\sqrt{23 / 3}$ )

$$
\widetilde{T}(z)=-\frac{1}{2} \partial \varphi_{L} \partial \varphi_{L}+\sqrt{\frac{23}{12}} \partial^{2} \varphi_{L}+\frac{1}{2} \partial X^{0} \partial X^{0}-\frac{1}{2} \partial X^{1} \partial X^{1}+2(\partial c) b+c \partial b .
$$

The general results of Ref. 13 concerning BRST cohomology for $d$ matter fields coupled to a Liouville field apply for the case when $d=2$. For the standard ghost number, and apart from some discrete states, the result is simple. The cohomology is isomorphic to a $(d-1)$ dimensional on-shell Fock space, and is therefore one-dimensional for our present case. While the results of Ref. [13] were given for the holomorphic sector, the total cohomology for the two sectors combined is just the tensor product, except for discrete states. Thus the Fock space must include both barred and unbarred oscillators. Letting $\left(p^{0}, p^{1}, p_{L}\right)$ denote the zero modes of $\left(X^{0}, X^{1}, \varphi_{L}\right)$, we can describe the counting of states in the cohomology as the set of states obtained by acting with a complete single set of oscillators $\left(a_{n}^{\dagger}, \bar{a}_{n}^{\dagger}\right)(n>0)$ on a vacuum $\left|p^{0}, p^{1}, p_{L}\right\rangle$, where the on-shell condition arising from the $\left(L_{0}+\bar{L}_{0}\right)=0$ constraint requires that

$$
-\left(p^{0}\right)^{2}+\left(p^{1}\right)^{2}+\left(p_{L}\right)^{2}+\frac{Q^{2}}{4}+N-2=0 .
$$

Here $N \geq 0$ is the total number operator of the oscillators that are acting on the vacuum state.

At first sight the field theoretical interpretation of the BRST cohomology seems encouraging since we saw that the free scalar field without gravity has precisely a Fock space that is generated with a single complete set of oscillators. The zero modes, however, do not seem to work out. In the case of one matter field with no gravity, the zero mode $p$ labeling the vacuum amounts to one single parameter. Here, in dilaton gravity, we have three zero modes $\left(p^{0}, p^{1}, p_{L}\right)$ and only one constraint, thus two parameters. This mismatch is generic, and will not disappear by adding extra matter fields. We therefore conclude that there is a difference between the naive semi-classical spectrum we would expect for a free scalar field and the spectrum we have obtained by BRST quantization of matter coupled dilaton gravity. (A string interpretation of the BRST physical states would correspond to a three dimensional target space and is not relevant to our purposes.) 
If this difference is truly significant, and remains so when the space is not a circle but an open line, one would reach the conclusion that there seems to be no known two-dimensional quantum gravity theory coupled to matter whose set of physical states resembles closely that of a matter theory without gravity. A possible exception to this conclusion might be the quantum gravity provided by two-dimensional string theory. In this case there is strong evidence that the semi-classical limit of this theory corresponds to a massless scalar field propagating on a particular two-dimensional space-time background [30].

\section{CONCLUSION AND DISCUSSION}

In this paper we have addressed questions relevant to the definition and quantization of two-dimensional dilaton gravity. One important issue concerns field redefinitions and the starting point for quantization. In Ref. [7] a series of field redefinitions, some not invertible, reduced the dilaton gravity theory without cosmological constant to a conformal theory of two free scalar fields with opposite norm. As we have seen, even the theory with nonzero cosmological term can be brought to the same final form, as was done in Ref. [6] and Section III] with the help of temporally local but spatially non-local field transformations. An obvious question is whether the physics is changed by such redefinitions, and the fact that the same final theory emerges, regardless whether a cosmological constant is present, suggests that field redefinitions are non-trivial in the quantum case. Perhaps the redefinitions ought to be interpreted as part of the definition of the quantum theory; namely, the classical system is first manipulated into a particular form, and then quantized.

After carrying out our reduction to indefinite quadratic form we have seen how pure dilaton gravity can be quantized without obstruction by adopting particular quantization scheme for the scalar with negative kinetic energy. As a byproduct, we found a unitary quantization of a string in two-dimensional Minkowski space where all Virasoro constraints are satisfied. There are only two physical states, and therefore this is not a rich spectrum. Nevertheless, as a matter of principle, it is quite surprising that there are nontrivial states annihilated by all the Virasoro operators, since in standard string theory the vacuum state 
and all physical states are only annihilated by the positively moded Virasoro operators. While our states have infinite norm they can be presented as well defined linear superposition of Fock space states.

Once matter is included, the theory is still of indefinite quadratic form, but the constraints cannot be solved owing to the quantal commutator anomaly. We emphasize that classically the constraints can be satisfied and the equations can be solved [6]. Also when the quantized matter comprises point particles, there are no serious obstructions to satisfying the constraints and the quantum theory can be analyzed to the end [32]. It is only quantized matter fields that produce problems. We have seen that progress can be made when various modifications or improvements are effected. While the obstructions can be overcome in various ways, the physical picture in the resulting quantum theory bears no resemblance to the classical physics, except in BRST quantization where there is a vague resemblance. This suggests that semi-classical analysis may be of questionable relevance even for an approximate description [33, 34].

It would be interesting to clarify the status of quantization of four-dimensional gravity in light of the observations made in the present work. Since physical gravity has propagating states, we would expect that the relevant constraints of the quantum theory will exhibit a center even in the absence of matter. If this center could be determined precisely one could investigate if it can be removed by introducing special variables, improvement terms, and/or by the use of alternative quantization schemes, as in the two-dimensional model.

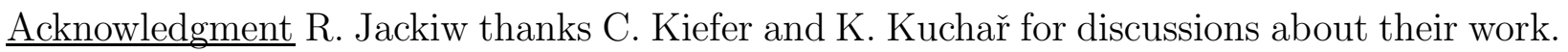




\section{REFERENCES}

[1] R. Jackiw, C. Teitelboim in Quantum Theory of Gravity, S. Christensen, ed. (A. Hilger, Bristol, UK, 1984).

[2] D. Cangemi, Phys. Lett. B297 (1992) 261;

A. Achúcarro, Phys. Rev. Lett. 70 (1993) 1037.

[3] A. Polyakov, Mod. Phys. Lett. A2 (1987) 893, Gauge Fields and Strings (Harwood, New York, NY, 1987);

D. Karakhanyan, R. Manvelyan and R. Mkrtchyan, Phys. Lett. B329 (1994) 185.

[4] P. Ginsparg and G. Moore, "Lectures on 2D Gravity and 2D String Theory", in TASI 1992: Recent Directions in Particle Physics, J. Harvey and J. Polchinski, eds. (World Scientific, Singapore, 1993).

[5] C. Callan, S. Giddings, J. Harvey, and A. Strominger, Phys. Rev. D 45 (1992) 1005;

H. Verlinde in Sixth Marcel Grossmann Meeting on General Relativity, M. Sato and T. Nakamura, eds. (World Scientific, Singapore, 1992).

[6] D. Cangemi and R. Jackiw, Phys. Lett. B337 (1994) 271.

[7] A. Bilal and C. Callan, Nucl. Phys. B394 (1993) 73;

S. P. de Alwis, Phys. Lett. B317 (1993) 46.

[8] This alternative derivation is due to E. Benedict, Ph.D. thesis, Boston University (unpublished).

[9] D. Cangemi and R. Jackiw, Phys. Rev. D 50 (1994) 3913;

D. Amati, S. Elitzur and E. Rabinovici, Nucl. Phys. B418 (1994) 45.

[10] K. Kuchař, Phys. Rev. D 39 (1989) 2263;

K. Kuchař and G. Torre, J. Math. Phys. 30 (1989) 1769.

[11] T. Fujiwara, Y. Igarashi, J. Kubo and T. Tabei, Phys. Lett. B336 (1994) 157.

[12] J. Goldstone, unpublished;

A. M. Polyakov, Mod. Phys. Lett. A6 (1991) 635;

B. Lian and G. Zuckerman, Phys. Lett. B254 (1991) 417; B266 (1991) 21;

P. Bouwknegt, J. McCarthy and K. Pilch, Comm. Math. Phys. 145 (1992) 541;

E. Witten and B. Zwiebach, Nucl. Phys. B377 (1992) 55.

[13] A. Bilal, Phys. Lett. B282 (1992) 309.

[14] D. Cangemi and R. Jackiw, Phys. Rev. Lett. 69 (1992) 233, Ann. Phys. (NY) 225 (1993) 229.

[15] K. Stelle and P. West, Phys. Rev. D 21 (1980) 1466.

[16] G. Grignani and G. Nardelli, Nucl. Phys. B412 (1994) 320.

[17] In fact, $q_{(0)}$ may also have a component along $I$. Such generalization would lead to a new and two-dimension specific interaction [6.14], which we do not consider in this paper.

[18] A coupling of the form $\sqrt{-g} \varphi_{3} R$ can also be incorporated in our formalism by adding $\mathcal{A} \epsilon^{\mu \nu}\left(\frac{1}{2} q^{a} \epsilon_{a b} \partial_{\mu} q^{b}-\left\langle q \mid A_{\mu}\right\rangle\right) \partial_{\nu} \varphi_{3}$ to the matter Lagrangian [6,14]. Such a term is not 
gauge invariant but its variation is a total derivative, hence the resulting action is gauge invariant. We do not include this interaction in the present discussion.

[19] D. Louis-Martinez, J. Gegenberg and G. Kunstatter, Phys. Lett. B321 (1994) 193;

T. Strobl, Phys. Rev. D 50 (1994) 7346.

[20] This commutator demonstrates that it is unnecessary to satisfy the momentum constraint (4.1b) separately, provided the energy constraint (4.1a), i.e. the Wheeler-DeWitt equation, is solved, since according to (4.3) the latter implies the former; see V. Moncrief and C. Teitelboim, Phys. Rev. D 6, 966 (1972).

[21] E. Benedict, Phys. Lett. B340 (1994) 43.

[22] This is reminiscent of other work, which showed that the Virasoro anomaly can be viewed as the curvature of a functional connection: M. Bowick and S. G. Rajeev, Phys. Rev. Lett. 58 (1987) 535 (E)1158, Nucl. Phys. B293(1987) 348 and B296 (1988) 1007. For similar observations about chiral anomalies, see e.g. A. Niemi and G. Semenoff, Phys. Lett. B175(1986) 439.

[23] Note that the functional derivative with respect to $X^{ \pm}$may be written in terms of one with respect to $X^{ \pm \prime}: \frac{\delta}{\delta X^{ \pm}(\sigma)}=-\partial_{\sigma} \frac{\delta}{\delta X^{ \pm \prime}(\sigma)}$. Therefore $X^{ \pm \prime}(\sigma)$ is the only gravity variable in $4.17 \mathrm{~b}$ ).

[24] That eliminating $P_{ \pm}$will expose interesting structures was suggested by D. Bak (private communication).

[25] M. Bos, Phys. Rev. D 34 (1986) 3750.

[26] Polyakov, Ref. 3.

[27] The observation is due to D. Bak (private communication).

[28] M. Bershadsky and H. Ooguri, Commun. Math. Phys. 126 (1989) 49.

[29] That the sign of a commutator anomaly depends on how the vacuum state is defined was first observed by P. Jordan, Z. Phys. 93 (1935) 464. In the context of the Virasoro anomaly, this point is explained by R. Floreanini and R. Jackiw, Phys. Lett. B175 (1986) 428.

[30] A pedagogical explanation of this point can be found in: J. Polchinski, "What is String Theory?", hep-th/9411028.

[31] F. David, Mod. Phys. Lett. A3 (1988) 1651;

J. Distler and H. Kawai, Nucl. Phys. B321 (1989) 509.

[32] D. Cangemi and R. Jackiw. Phys. Rev. D 50 (1994) 3913;

D. Bak and D. Seminara, "String-Inspired Gravity with Interacting Point-Particles," MIT preprint MIT-CTP-2431, June 1995.

[33] D. Giulini and C. Kiefer, Class. Quantum Grav. 12 (1995) 403.

[34] E. Keski-Vakkuri, G. Lifschytz, S. Mathur, and M. Ortiz, Phys. Rev. D (in press); G. Lifschytz, S. Mathur and M. Ortiz, "A Note on the Semi-Classical Approximation in Quantum Gravity", MIT preprint MIT-CTP-2384, Nov. 1994, gr-qc/9412040. 